\title{
SOME PROPERTIES OF MEASURE AND CATEGORY
}

\author{
BY \\ ARNOLD W. MILLER ${ }^{1}$
}

\begin{abstract}
Several elementary cardinal properties of measure and category on the real line are studied. For example, one property is that every set of real numbers of cardinality less than the continuum has measure zero. All of the properties are true if the continuum hypothesis is assumed. Several of the properties are shown to be connected with the properties of the set of functions from integers to integers partially ordered by eventual dominance. Several, but not all, combinations of these properties are shown to be consistent with the usual axioms of set theory. The main technique used is iterated forcing.
\end{abstract}

Six properties of measure and category on the real line are studied. $\mathbf{A}(c)$ is the proposition that the union of fewer than continuum many meager sets is meager. $\mathrm{B}(c)$ says that the real line is not the union of fewer than continuum many meager sets. $U(c)$ is the proposition that every subset of the real line of cardinality less than continuum is meager. $\mathrm{A}(m), \mathrm{B}(m)$, and $\mathrm{U}(m)$ are defined analogously by replacing meager by measure zero. In the first section some equivalent forms of these properties are given, for example, it is shown that $\mathrm{A}(c)$ iff $\mathrm{B}(c)$ and every family of elements of $\omega^{\omega}$ of cardinality less than the continuum is eventually dominated by an element of $\omega^{\omega}$. Characterizations of $\mathrm{U}(c)$ and $\mathrm{B}(c)$ are also given. In the second section we prove some theorems about unions of closed sets of measure zero, small dominating families, and strong measure zero sets. In the remaining sections several combinations of these properties are shown to be consistent with ZFC. These consistency results are summarized in the third section. The last section contains some open problems. I would like to thank K. Kunen for several helpful discussions.

1. The properties and some of their equivalent forms. All the properties we consider are equivalent whether stated for $2^{\omega}, \omega^{\omega}$, or the real line. For definiteness they will be stated for the Cantor space $2^{\omega}$, so we will begin by reviewing the usual product topology and measure on $2^{\omega}$ and also establish some standard terminology. For sets $X$ and $Y$ let $Y^{X}$ denote the set of functions from $X$ into $Y$ and $|X|$ denote cardinality of $X$. Let $2^{<\omega}=\cup\left\{2^{n}: n<\omega\right\}$ and similarly $\omega^{<\omega}$. Note that for $s \in 2^{<\omega},|s|$ is the length of $s$ when thought of as a sequence of zeros and ones. For $s \in 2^{<\omega}$ let $[s]=\left\{x \in 2^{\omega}: s \subseteq x(x\right.$ extends $\left.s)\right\}$, then the usual product topology on $2^{\omega}$ is given by taking $\left\{[s]: s \in 2^{<\omega}\right\}$ as a basis for the open sets and the usual product measure $\mu$ is given by letting $\mu([s])=2^{-|s|}$. For $s$ and $t$ finite sequences let

Received by the editors July 25, 1979.

AMS (MOS) subject classifications (1970). Primary 02K05; Secondary 54A25.

${ }^{1}$ Research partially supported by an NSF grant. 
$s \frown t$ denote the concatenation of $s$ and $t$. Let $[X]^{<\omega}$ and $[X]^{\omega}$ denote the set of finite subsets of $X$ and the set of countably infinite subsets of $X$ respectively. Let $2^{<n}=\cup\left\{2^{m}: m \leqslant n\right\}$ and for $n, m \in \omega$ let $[n, m)=\{i \in \omega: n \leqslant i<m\}$. The symbols " $\exists^{\infty}$ " and " $\forall$ " abbreviate "there exist infinitely many" and "for all but finitely many" respectively. Recall that a set is nowhere dense iff its closure has no interior; and a set is meager (equivalently first category) iff it is the countable union of nowhere dense sets.

We consider the following three properties each applied to category and measure. The strongest property, Additivity $(A(c))$, says that the union of less than $\left|2^{\omega}\right|$ meager sets is meager. The next property, Baire $(\mathrm{B}(c))$, says that the union of less than $\left|2^{\omega}\right|$ meager sets is not $2^{\omega}$. Finally the last property, Uniformity $(\mathrm{U}(c))$, says that every $X \subseteq 2^{\omega}$ of cardinality less than $\left|2^{\omega}\right|$ is meager. $\mathrm{A}(m), \mathrm{B}(m)$, and $\mathrm{U}(m)$ are defined analogously by replacing meager by measure zero. These properties are considered (but not named) in [MS] where the basic facts about the Cohen real extension and the Solovay real (random real) extension are noted (but not proved, these proofs will appear in the survey paper [K1]). Note that trivially $\mathbf{A}(c)$ implies $\mathrm{B}(c)$ and $\mathrm{U}(c)$; and $\mathrm{A}(m)$ implies $\mathrm{B}(m)$ and $\mathrm{U}(m)$. The only other implications known are Theorem 1.1(a) and (b) which are due to Rothberger [R1]. This leaves fourteen possible combinations (see the chart in $\$ 3$ which is taken from [K1]).

THEOREM 1.1. (a) $\mathrm{B}(m)$ implies $\mathrm{U}(c)$.

(b) $\mathrm{B}(c)$ implies $\mathrm{U}(m)$.

Proof. For $x, y \in 2^{\omega}$ let $x+y$ be the pointwise sum modulo 2 of $x$ and $y$. Let $G$ be a comeager measure zero subset of $2^{\omega}$ (see [O]). If $X \subseteq 2^{\omega}$ is not meager, then $2^{\omega}=\cup\{x+G: x \in X\}$. This is because if $z \in 2^{\omega}-\cup\{x+G: x \in X\}$, then $(z+G) \cap X=\varnothing$ but $z+G$ is comeager, contradicting the fact that $X$ is not meager. This proves the contrapositive of (a); and (b) is proved similarly.

For $f, g \in \omega^{\omega}$ define $f<g$ iff $\forall^{\infty} m f(m)<g(m)$ (that is $g$ eventually dominates $f$ ). Let $\mathrm{D}$ stand for the property that $\forall F \subseteq \omega^{\omega}$ if $|F|<\left|\omega^{\omega}\right|$ then $\exists f \in \omega^{\omega} \forall g \in F$ $f<g$. D says that every family of small cardinality is dominated. Rothberger [R2] noted that $\mathrm{D}$ implies $\mathrm{U}(c)$. This is because for any $f \in \omega^{\omega},\left\{g \in \omega^{\omega}: g<f\right\}$ is meager. Truss (see $[\mathbf{T}]$ ) showed that $\mathrm{B}(c)+\mathrm{D}$ implies $\mathrm{A}(c)$. We prove the converse.

THEOREM 1.2. $\mathrm{A}(c)$ iff $\mathrm{B}(c)+\mathrm{D}$.

Proof. Suppose $F \subseteq \omega^{\omega},|F|<\left|\omega^{\omega}\right|$, and no $f \in \omega^{\omega}$ eventually dominates every $g \in F$. We assume every $g$ in $F$ is strictly increasing and define $n_{k}$ for $k<\omega$ by letting $n_{k+1}=g\left(n_{k}\right)$, and define $C(g)=\left\{h \in 2^{\omega}: \forall k h\left(n_{k}\right)=1\right\}$. Note that each $C(g)$ is closed nowhere dense in $2^{\omega}$.

Claim. $\cup\{C(g): g \in F\}$ is not meager.

Proof. Let $\left\{C_{n}: n<\omega\right\}$ be a countable family of closed nowhere dense sets. Define $m_{k}<\omega$ for $k<\omega$ as follows. Let $m_{k+1}>m_{k}$ be such that $\forall i<k, \forall s \in$ $2^{m_{k}}, \exists t \in 2^{m_{k+1}}, t \supseteq s$ and $[t] \cap C_{i}=\varnothing$. Let $Z=\left\{m_{k}: k<\omega\right\}$. Then there is a $g \in F$ with the property that for its associated sequence $\left\langle n_{k}: k\langle\omega\rangle\right.$ there are infinitely many $k$ such that

$$
\left|\left[n_{k}, n_{k+1}\right) \cap Z\right| \geqslant 2 \text { where }\left[n_{k}, n_{k+1}\right)=\left\{m<\omega: n_{k} \leqslant m<n_{k+1}\right\} .
$$


This is true because if not, then define $f(k)=m_{k}$ for all $k$ and let $\hat{f}$ eventually dominate the tails of $f$, i.e. $\forall k<\omega$ if $h$ is defined by $h(n)=f(n+k)$ for all $n$ then $h<\hat{f}$. For any $g \in F$ and its associated $n_{k}$ 's $\exists l \forall k>l,\left|\left(n_{k}, n_{k+1}\right) \cap Z\right|<1$. Let $m_{\hat{k}}$ be the least element of $\left[n_{l}, \infty\right) \cap Z$ and then $\forall k>l, g(k)<n_{k}<m_{\hat{k}+k}=$ $f(\hat{k}+k)$ and thus $g<\hat{f}$. This contradicts the fact that nothing dominates $F$. Letting $g \in F$ be such that there are infinitely many $k$ such that $\left|\left[n_{k}, n_{k+1}\right) \cap Z\right|>$ 2, it follows easily from the definition of $Z$ that each $C_{n}$ is nowhere dense in $C(g)$ (the relative topology), and thus $\cup\{C(g): g \in F\}$ is not included in $\cup\left\{C_{n}: n<\right.$ $\omega\}$. Since the $C_{n}$ 's were arbitrary to start with the claim is proved and so is the theorem.

Note that $\mathrm{B}(c)$ is necessary in Theorem 1.2 since $\mathrm{D}+\neg \mathrm{A}(c)$ is true in the Mathias real model (see §6). The following theorem characterizes $\mathrm{U}(c)$ (every $X \subseteq 2^{\omega}$ with $|X|<\left|2^{\omega}\right|$ is meager).

THEOREM 1.3. $\mathrm{U}(c)$ iff $\forall F \subseteq \omega^{\omega}$ if $|F|<\left|\omega^{\omega}\right|$ then $\exists X \subseteq \omega$ infinite, $\exists f \in \omega^{\omega}$, $\forall g \in F, \forall{ }^{\infty} n \in X, f(n) \neq g(n)$ (that is $f$ is eventually different on $X$ from every element of $F$ ).

Proof. $(\Leftarrow)$ This is easy since for any $f$ and $X\left\{g: \forall^{\infty} n \in X, f(n) \neq g(n)\right\}$ is meager.

$\Leftrightarrow$ Suppose not and let $\kappa=\left|2^{\omega}\right|^{++}$and choose $M$ an elementary substructure of $(H(\kappa), \varepsilon)(H(\kappa)$ is the set of hereditarily of cardinality less than $\kappa$ sets) with the properties $|M|<\left|2^{\omega}\right|$ and $\forall f \in \omega^{\omega} \forall X \subseteq \omega$ infinite $\exists g \in M \cap \omega^{\omega}, \exists^{\infty} n \in X$, $f(n)=g(n)$. We show $M \cap 2^{\omega}$ is not meager in $2^{\omega}$.

Claim. $\forall g \in \omega^{\omega}, \exists\left\langle n_{k}: k<\omega\right\rangle \in M$ strictly increasing and $\exists^{\infty} k, g\left(n_{k}\right)<n_{k+1}$.

Proof. We may as well assume $g$ is strictly increasing. Choose $f \in \omega^{\omega} \cap M$ such that $\exists^{\infty} k, f(k)=g(k)$. Build in $M,\left\langle m_{k}: k<\omega\right\rangle$ so that $\forall k \forall i \leqslant m_{k}, f(i)<m_{k+1}$. Let $X=\{i: f(i)=g(i)\}$. Assume $\cup\left\{\left[m_{k}, m_{k+1}\right) \cap X: k\right.$ even $\}$ is infinite, then $n_{k}=m_{2 k}$ works since if $i \in\left[m_{2 k}, m_{2 k+1}\right) \cap X$ then $g\left(n_{k}\right)=g\left(m_{2 k}\right)<g(i)=f(i)$ $<m_{2 k+2}=n_{k+1}$. A similar argument works if $\bigcup\left\{\left[m_{k}, m_{k+1}\right) \cap X: k\right.$ odd $\}$ is infinite.

Now suppose $C_{n}$ for $n<\omega$ are closed nowhere dense in $2^{\omega}$ and $C_{n} \subseteq C_{n+1}$ for all $n$. Define $f: \omega \rightarrow 2^{<\omega}$ so that $\forall n<\omega \forall s \in 2^{<n}[s \frown f(n)] \cap C_{n}=\varnothing$. By the claim $\exists\left\langle n_{k}: k\langle\omega\rangle \in M\right.$ such that $n_{k+1}>k \cdot n_{k}$ for all $k$ and $\exists^{\infty} k\left|f\left(n_{k}\right)\right|<n_{k+1}$. Let $X=\left\{n_{k}: k\right.$ even and $\left.\left|f\left(n_{k}\right)\right|<n_{k+1}\right\}$. By hypothesis on $M \exists g: \omega \rightarrow 2^{<\omega} g \in M$ and $\exists^{\infty} k \in X \quad g\left(n_{k}\right)=f\left(n_{k}\right)$. We can assume $\forall k\left|g\left(n_{k}\right)\right|<n_{k+1}$. Let $z=$ $g\left(n_{0}\right) \frown g\left(n_{2}\right) \frown g\left(n_{4}\right) \frown \cdots ;$ then $z \in M \cap 2^{\omega}$ and $z \notin \cup\left\{C_{n}: n<\omega\right\}$, since if $g\left(n_{2 k}\right)=f\left(n_{2 k}\right)$ then $\left|g\left(n_{0}\right) \frown g\left(n_{2}\right) \frown \ldots \frown g\left(n_{2 k-2}\right)\right|<(2 k-2) \cdot n_{2 k-1}<n_{2 k}$ and therefore $z \notin C_{n_{2 k}}$.

REMARK. The $\sigma$-ideal $I$ generated by sets of the form

$$
\left\{g \in \omega^{\omega}: \forall \forall^{\infty} \in X, g(n) \neq f(n)\right\}
$$

for $X \subseteq \omega$ infinite and $f \in \omega^{\omega}$ is a proper subset of the ideal of meager subsets. To see this let $\left\langle s_{n}: n\langle\omega\rangle\right.$ enumerate $\omega^{<\omega}$ without repetitions and let $C=\{x \in$ $\left.\omega^{\omega}: \neg \exists n<\omega s_{n} \frown(n) \subseteq x\right\}$. It is easily checked that $C$ is closed nowhere dense in $\omega^{\omega}$. To see that $C \notin I$ let $f_{n}, X_{n}$ for $n<\omega$ be given. Build a sequence $t_{m} \subseteq t_{m+1}$ of 
elements of $\omega^{<\omega}$ so that $\cup\left\{t_{m}: m<\omega\right\}=z \in C$ but $\forall n \exists^{\infty} m \in X_{n} f_{n}(m)=$ $z(m)$. This can be done since given $t \in \omega^{<\omega}$ such that $[t] \cap C \neq \varnothing$ and $n<\omega$ we can choose $l \in X_{n}$ such that $l>|t|+1$; then $T=\{r: r \supseteq t \wedge|r|=l \wedge[r] \cap C$ $\neq \varnothing\}$ is infinite. So $\exists r \in T$ such that $\left[r \frown\left(f_{n}(l)\right)\right] \cap C \neq \varnothing$.

Next we characterize $\mathrm{B}(c)$.

TheOREM 1.4. $\mathrm{B}(c)$ iff $\left[\forall F \subseteq \omega^{\omega}\right.$ if $|F|<\left|\omega^{\omega}\right|$ then $\exists f \in \omega^{\omega} \forall g \in F \quad \exists^{\infty} n$ $(f(n)=g(n) \wedge \forall i<n f(i)<n)]$.

Proof. $(\Rightarrow)$ It is easily checked that for any $g \in \omega^{\omega}\left\{f \in \omega^{\omega}: \exists^{\infty} n(f(n)=g(n)\right.$ $\wedge \forall i<n f(i)<n)\}$ is a comeager $G_{\delta}$ subset of $\omega^{\omega}$.

$(\Leftarrow)$ Let $M$ be any elementary substructure of $(H(\kappa), \varepsilon)$ such that $\kappa>\left|2^{\omega}\right|^{++}$and $|M|<\left|2^{\omega}\right|$. By hypothesis $\exists f \in \omega^{\omega} \forall g \in M \cap \omega^{\omega} \exists^{\infty} n(f(n)=g(n) \wedge \forall i<n$ $f(i)<n)$. Let $\left\langle s_{n}: n\langle\omega\rangle \in M\right.$, list $2^{<\omega}$, and define $\left.z=s_{f(0)}\right\urcorner s_{f(1)} \frown s_{f(2)} \frown \cdots$.

Claim. $\forall C \subseteq 2^{\omega}$ closed nowhere dense, if $C \in M$ then $z \notin C$.

Proof. Define $g \in \omega^{\omega} \cap M$ by $g(n)$ is the least $m<\omega$ such that $\forall i_{0}, \ldots, i_{n-1}$ $<n\left[s_{i_{0}} \frown s_{i_{1}} \frown \ldots \frown s_{i_{n-1}} \frown s_{m}\right] \cap C \neq \varnothing$. If $g(n)=f(n) \wedge \forall i<n f(i)<n$ then $z \notin C$. This proves the claim and since $M$ was arbitrary it proves the theorem.

2. Closed sets of measure zero, small dominating families, and strong measure zero sets. It is well known that $\mathrm{B}(c)$ does not imply $\mathrm{B}(m)$ (Cohen real model, see [K1]), however,

TheOREM 2.1. B(c) implies the union of fewer than $\left|2^{\omega}\right|$ closed sets of measure zero has measure zero.

Proof. Let $C_{n}=\left\{D_{m}^{n}: m<\omega\right\}=\left\{D \subseteq 2^{\omega}: D\right.$ clopen $\left.\wedge \mu(D)<1 / 2^{n}\right\}$. Let $E_{\alpha} \subseteq 2^{\omega}$ for $\alpha<\lambda$ be closed sets of measure zero. Then $G_{\alpha}=\left\{f \in \omega^{\omega}: \exists^{\infty} n\right.$ $\left.E_{\alpha} \subseteq D_{f(n)}^{n}\right\}$ is a comeager $G_{\delta}$ set. If $f \in \cap\left\{G_{\alpha}: \alpha<\lambda\right\}$ then

$$
\mu\left(\bigcap_{n<\omega} \bigcup_{m>n} D_{f(m)}^{m}\right)=0 \text { and } \forall \alpha<\lambda E_{\alpha} \subseteq \bigcap_{n<\omega} \bigcup_{m>n} D_{f(m)}^{m} \text {. }
$$

SD says that $\exists F \subseteq \omega^{\omega}|F|<\left|\omega^{\omega}\right|$ and $\forall f \in \omega^{\omega} \exists g \in F f<g$ (i.e. there is a small dominating family).

THEOREM 2.2. SD implies there is a union of fewer than $\left|2^{\omega}\right|$ closed sets of measure zero that does not have measure zero.

Proof. Let $M \prec(H(\kappa), \varepsilon)$ for some $\kappa \geqslant\left|2^{\omega}\right|^{++}$and $|M|<\left|2^{\omega}\right|$ and $F \subseteq M$, where $F$ witnesses SD. We show that $X=\cup\left\{C \subseteq 2^{\omega}: C\right.$ closed measure zero $\wedge$ $C \in M\}$ does not have measure zero. Suppose it does and let $H \subseteq 2^{\omega}$ be an open set with $\mu(H)<\frac{1}{4}$ and $X \subseteq H$.

Claim. $\forall X \subseteq \omega$ infinite $\exists\left\langle n_{k}, k<\omega\right\rangle \in M$ so that $0=n_{0}$ and $\forall k n_{k}<n_{k+1}$ and $\left|X \cap\left[n_{k}, n_{k+1}\right)\right| \geqslant 2$.

Proof. Choose $f(n)$ for all $n$ so that $|[n, f(n)) \cap X|>2$. Let $g \in \omega^{\omega} \cap M$ be strictly increasing and $\forall n f(n)<g(n)$, and let $n_{k+1}=g\left(n_{k}\right)$ for all $k>0\left(n_{0}=0\right)$.

Build a sequence $n_{k}<n_{k+1}$ for $k<\omega$ with $n_{0}=0$ and so that $\forall s \in 2^{<n_{k}}$

$$
\mu\left((H \cap[s])-\bigcup\left\{[t]: t \supseteq s, t \in 2^{n_{k+1}}, \text { and }[t] \subseteq H\right\}\right)<\frac{1}{2^{n_{k}+3}} .
$$


By the claim we may assume $\left\langle n_{k}: k\langle\omega\rangle \in M . \forall k<\omega\right.$ let $D_{k}=\left\{n_{1}, \ldots, n_{k}\right\}$ and let $s_{k}: D \rightarrow 2$ be constantly 1 . Define $C=\cap\left\{\left[s_{k}\right]: k<\omega\right\}$ and note that $C \in M$ is a closed set of measure zero. We will show $C \nsubseteq H$ by showing in effect that the "measure" of $H$ with respect to $C$ is less than $\frac{1}{2}$. For any $k<\omega$ let $\varepsilon_{k}=1 / 2^{2 n_{k}+3}$ and let

$$
U_{k}=\bigcup\left\{[t]: t \supseteq s_{k} \wedge t \in 2^{n_{k+1}} \wedge[t] \subseteq H\right\} .
$$

Then $\mu\left(H \cap\left[s_{k+1}\right]\right)=\mu\left(\left(H-U_{k}\right) \cap\left[s_{k+1}\right]\right)+\mu\left(U_{k} \cap\left[s_{k+1}\right]\right)$. By our choice of $n_{k+1}, \mu\left(H-U_{k} \cap\left[s_{k}\right]\right)<\varepsilon_{k}$. Since $U_{k}$ lives in $2^{n_{k+1}}, \mu\left(U \cap\left[s_{k+1}\right]\right)=\frac{1}{2} \mu\left(U \cap\left[s_{k}\right]\right)$ and since $U_{k} \subseteq H \cap\left[s_{k}\right], \mu\left(H \cap\left[s_{k+1}\right]\right) \leqslant \frac{1}{2} \mu\left(H \cap\left[s_{k}\right]\right)+\varepsilon_{k}$. By induction on $k$ it is easily shown that

$$
\mu\left(H \cap\left[s_{k+1}\right]\right) \leqslant \frac{1}{2^{k+1}} \mu(H)+\sum_{l=0}^{k} \frac{1}{2^{k-l}} \varepsilon_{l} .
$$

And thus

$$
2^{k+1} \mu\left(H \cap\left[s_{k+1}\right]\right) \leqslant \mu(H)+\sum_{l=0}^{k} 2^{l+1} \varepsilon_{l}
$$

Since $n_{l} \geqslant l, 2^{l+1} \varepsilon_{l}=2^{l+1} / 2^{2 n_{l}+4} \leqslant 1 / 2^{l+3}$ and thus for all $k$

$$
2^{k} \mu\left(H \cap\left[s_{k}\right]\right)<\frac{1}{2} \text {. }
$$

Define the canonical homeomorphism $F$ from $2^{\omega}$ onto $C$ as follows:

$$
F(X)(l)= \begin{cases}1, & \text { if } l=n_{k} \text { for some } k>0, \\ X(l-k), & \text { if } k+1 \text { is the least integer such that } l<n_{k+1}\end{cases}
$$

Consider the Borel measure $\mu^{*}$ defined by $\mu^{*}(X)=\mu\left(F^{-1}(X)\right)$. Let

$$
H_{k}=\bigcup\left\{[s]: s \in 2^{n_{k+1}} \wedge[s] \subseteq H\right\} .
$$

Note that $\forall t \in 2^{n_{k+1}} \mu^{*}([t]) \leqslant 1 / 2^{n_{k+1}-k}=2^{k} \mu([t])$. And therefore

$$
\mu^{*}\left(H_{k}\right)=\mu^{*}\left(H_{k} \cap\left[s_{k}\right]\right) \leqslant 2^{k} \mu\left(H_{k} \cap\left[s_{k}\right]\right)<2^{k} \mu\left(H \cap\left[s_{k}\right]\right)<\frac{1}{2} .
$$

Therefore $\mu^{*}(H)=\lim \mu^{*}\left(H_{k}\right) \leqslant \frac{1}{2}$ and thus $C-H \neq \varnothing$.

For $X \subseteq 2^{\omega}$ we say that $X$ has strong measure zero iff $\forall f \in \omega^{\omega} \exists s_{n} \in 2^{f(n)}$ for $n<\omega$ such that $X \subseteq \cup\left\{\left[s_{n}\right]: n<\omega\right\}$. Let SMZ stand for the proposition $\forall X \subseteq$ $2^{\omega}$ if $|X|<\left|2^{\omega}\right|$ then $X$ has strong measure zero. For $B \subseteq \omega^{\omega}, B$ is bounded iff $\exists f \in \omega^{\omega} \forall g \in B \forall n<\omega g(n)<f(n)$. The following theorem is essentially proven in [R2] and [R6].

TheOREM 2.3. SMZ iff $\forall E \subseteq \omega^{\omega}$ if $|E|<\left|\omega^{\omega}\right|$ and $E$ is bounded then $\exists f \in \omega^{\omega}$ $\forall h \in E \exists^{\infty} n f(n)=h(n)$.

Proof. $(\Leftarrow)$ Suppose $X \subseteq 2^{\omega},|X|<\left|2^{\omega}\right|$, and $f \in \omega^{\omega}$. For every $x \in X$ let $g_{x}: \omega \rightarrow 2^{<\omega}$ be defined by $g_{x}(n)=x \uparrow f(n)$. Let $E=\left\{g_{x}: x \in X\right\}$ and note that $E$ corresponds to a bounded set in $\omega^{\omega}$ since $\forall n \forall x g_{x}(n)$ lies in a fixed set of size $2^{f(n)}$. Thus $\exists h: \omega \rightarrow 2^{<\omega} \forall n<\omega h(n) \in 2^{f(n)}$ and $\forall g \in E \exists^{\infty} n h(n)=g(n)$ and this implies $X \subseteq \cup\{[h(n)]: n<\omega\}$. 
$(\Rightarrow)$ Assume without loss of generality that $E \subseteq\left\{f: \omega \rightarrow 2^{<\omega}: \forall n f(n) \in 2^{g(n)}\right\}$ $=Y$ for some $g \in \omega^{\omega}$ and $\forall f, f^{\prime} \in Y$ if $\forall^{\infty} n f(n)=f^{\prime}(n)$ then $f \in E$ iff $f^{\prime} \in E$. Define $c: E \rightarrow 2^{\omega}$ by $c(f)=f(0) \frown f(1) \frown f(2) \frown \ldots$ (the infinite concatenation of $f)$. For every $n<\omega$ let $f(n)=\Sigma\{g(m): m \leqslant n\}$. Since $c(E)$ has strong measure zero $\exists s_{n} \in 2^{f(n)}$ such that $c(E) \subseteq \cup\left\{\left[s_{n}\right]: n<\omega\right\}$. For each $n<\omega$ let $h(n) \in 2^{g(n)}$ be defined by $h(n)(i)=s_{n}(f(n)-g(n)+i)$ (i.e. $h(n)$ is the last $g(n)$ digits of $\left.s_{n}\right)$. For any $f \in E \exists n x_{f} \in\left[s_{n}\right]$ which implies that $f(n)=h(n)$, since $E$ is closed under finite changes $\exists^{\infty} n f(n)=h(n)$.

ReMARKs. (1) Let $f: 2^{\omega} \rightarrow[0,1]$ be defined by $f(x)=\Sigma\left\{x(n) / 2^{n+1}: n<\omega\right\}$; then $f$ is onto and at worst two to one. Furthermore it is easily checked that if $f(X)=Y$ then $X$ has strong measure zero iff $Y$ has strong measure zero in the usual sense, i.e. given $\varepsilon_{n}>0$ for $n<\omega, \exists I_{n}$ intervals such that $Y \subseteq \cup\left\{I_{n}: n<\right.$ $\omega$ ) and the length of $I_{n}$ is less than $\varepsilon_{n}$.

(2) The assumption that $E$ is bounded in Theorem 2.3 can be seen to be necessary because of the model constructed in $\$ 7\left(\omega_{2}\right.$ infinitely often equal reals side by side).

(3) The $\omega_{2}$ Mathias reals model (\$6) shows that $\mathrm{SMZ}$ is not equivalent to $\mathrm{U}(m)$ (since Laver [L] shows that SMZ fails there).

(4) It is not hard to show that assuming $\mathrm{D}, \mathrm{B}(c)$ iff $\forall E \subseteq \omega^{\omega}$ if $|E|<\left|\omega^{\omega}\right|$ then $\exists f \in \omega^{\omega} \forall g \in E \exists^{\infty} n f(n)=g(n)$. Thus A(c) iff SMZ and D.

3. Summary of consistency results. This chart (from [K1]) summarizes the known consistency results for the six properties. The shaded boxes are eliminated by Rothberger's theorem.

We review here some standard terminology and facts about iterated forcing to be used in the remaining sections. It will be assumed that the reader is familiar with the method of unramified forcing as presented in Shoenfield [Sh] or Kunen [K2]. By $\Vdash$ " $\theta$ " is meant that $\forall p \in \mathbf{P} p \Vdash$ " $\theta$ ". Frequent use is made of the maximum principal, so that $\Vdash$ " $\theta$ " may be assumed instead of for some $p \in \mathbf{P}, p \Vdash$ " $\theta$ ". $M$ and $N$ will be used to stand for countable transitive models of $\mathrm{ZFC}+\mathrm{GCH}$. The assumption of $\mathrm{GCH}$ is of course not necessary in the case of c.c.c. extensions nor is it necessary that in the generic extension $\left|2^{\omega}\right|=\omega_{2}$. For simplicity we assume GCH in our ground model and $\left|2^{\omega}\right|=\left|2^{\omega_{1}}\right|=\omega_{2}$ holds in our extensions (except §8).

Two types of iteration will be employed-finite support and countable support. In general we will have a sequence of partial orders $\mathbf{P}_{\alpha}$ for $\alpha<\omega_{2}$ with $\mathbf{P}_{\alpha} \subseteq \mathbf{P}_{\beta}$ for $\alpha \leqslant \beta$ and $\mathbf{P}_{\delta}=\bigcup\left\{\mathbf{P}_{\alpha}: \alpha<\delta\right\}$ for limit $\delta$. For each $\alpha<\omega_{2}$ every $p \in \mathbf{P}_{\alpha}$ will be a map from $\omega_{2}$ into $M$ such that $\operatorname{supp}(p)=\{\beta: p(\beta) \neq 1\} \subseteq \alpha$ (the support of $p$ ) and $\forall \beta<\alpha p \uparrow \beta \Vdash$ " $p(\beta) \in \mathbf{P}^{\beta}$ " where $\mathbf{P}^{\beta}$ is some term for a partial order in the forcing language of $\mathbf{P}_{\beta}$. ( $p \uparrow \beta$ is the condition agreeing with $p$ up to $\beta$ and equal to 1 from then on.) For $G, \mathbf{P}^{\omega_{2}}$-generic over $M$, and $\alpha \leqslant \omega_{2}$ let $G_{\alpha}=G \cap \mathbf{P}_{\alpha}$. (For background see [K2, Chapter 8]).

In $\S \S 4$ and 5 supports will be finite and thus the partial orders iterated must have c.c.c. (if not $\omega_{1}$ is collapsed). It is assumed that the reader is familiar with the connection between Cohen reals and random (Solovay) reals, and measure and 
category (see [K1] and [So]). Thus the reason the category extension (forcing with $\mathbf{C}\left(\omega_{2}\right)=\left\{p \mid p: D \rightarrow 2 \wedge D \subseteq \omega_{2}\right.$ finite $\}$ ) satisfies $\mathrm{B}(c)+\neg \mathrm{U}(c)$ (and thus by Rothberger's theorem $\neg \mathrm{B}(m)+\mathrm{U}(m))$ is that the first $\omega_{1}$ Cohen reals $\left(\left\{x_{\alpha}: \alpha<\right.\right.$ $\left.\omega_{1}\right\}$ where $x_{\alpha}(n)=G(\alpha+n)$ for $n<\omega$ when $G$ is $\mathbf{C}\left(\omega_{2}\right)$-generic over $\left.M\right)$ are not meager (so $\neg \mathrm{U}(c)$ ) and also the $\omega_{2}$ Cohen reals cannot be covered by $\omega_{2}$ meager sets (so $\mathrm{B}(c)$ ). Showing this essentially boils down to the product lemma and the fact that $x \in 2^{\omega}$ is Cohen over $M$ iff it avoids meager sets coded in $M$.

\begin{tabular}{|c|c|c|c|c|c|c|c|}
\hline \multirow[b]{2}{*}{ measure } & \multirow{2}{*}{ category } & Add & $\mathrm{T}$ & F & $F$ & F & $F$ \\
\hline & & Baire & $\mathrm{T}$ & $\mathrm{T}$ & $F$ & $\mathrm{~T}$ & F \\
\hline Add & Baire & Unif & $\mathrm{T}$ & $\mathrm{T}$ & $\mathrm{T}$ & $F$ & F \\
\hline $\mathrm{T}$ & $\mathrm{T}$ & $\mathrm{T}$ & $\begin{array}{c}\mathrm{MA} \\
{[\mathrm{MS}]}\end{array}$ & ? & ? & Estäe & IIIIIIIIA \\
\hline$F$ & $\mathrm{~T}$ & T & $\begin{array}{l}\text { See } \\
\text { Conjecture } \\
\$ 9 \text { (2) }\end{array}$ & $\begin{array}{l}\text { Iterated } \\
\text { random } \\
\text { reals } \\
\$ 4\end{array}$ & $\begin{array}{l}\text { Infinitely } \\
\text { equal and } \\
\text { random } \\
\text { reals } \\
\$ 7\end{array}$ & Enyly & 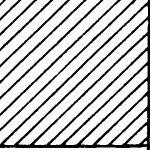 \\
\hline F & $\mathbf{F}$ & $\mathrm{T}$ & $\begin{array}{l}\text { Dominating } \\
\text { reals } \\
\$ 5\end{array}$ & $\begin{array}{l}\text { Eventually } \\
\text { different } \\
\text { reals } \$ 5\end{array}$ & $\begin{array}{c}\text { Mathias } \\
\text { reals } \\
\$ 6\end{array}$ & $\begin{array}{l}\text { Cohen } \\
\text { reals } \\
{[\mathrm{Kl}]}\end{array}$ & $\begin{array}{l}\text { Infinitely } \\
\text { equal reals } \\
\quad \$ 7\end{array}$ \\
\hline$F$ & $\mathrm{~T}$ & F & Ely" & W" & $\begin{array}{l}\text { Random } \\
\text { reals } \\
{[\mathrm{K} 1]}\end{array}$ & 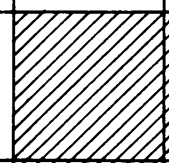 & 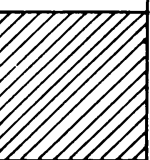 \\
\hline $\mathbf{F}$ & F & $F$ & Ely"ly & 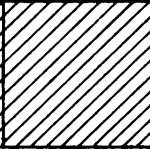 & $\begin{array}{l}\text { See } \\
\text { conjecture } \\
\$ 9 \text { (3) }\end{array}$ & \&alla & $\begin{array}{l}\text { Sllver or } \\
\text { Sack's } \\
\text { reals } \\
\$ 7\end{array}$ \\
\hline
\end{tabular}

In $\$ \S 6$ and 7 countable support will be used (also one side by side countable support). Here it will be assumed that the reader is familiar with [L] or [Ba]. See also [M1] and [BL].

In $\$ 8$ some partial results are given concerning the associated cardinals. One well known (but unpublished) fact which we will use there and in $\$ 5$ is

LEMMA. Suppose $\mathbf{P} \subseteq \mathbf{Q}$ are partial orders in $M$. Then the following are equivalent.

(A) $\forall G$, Q-generic over $M, G \cap \mathbf{P}$ is $\mathbf{P}$-generic over $M$.

(B) $\forall D \subseteq \mathbf{P}, D \in M$ dense in $\mathbf{P}, E=\{q \in \mathbf{Q}: \exists p \in D, q<p\}$ is dense in $\mathbf{Q}$.

Two conditions in $\mathbf{P}$ are compatible in $\mathbf{P}$ iff they are compatible in $\mathbf{Q}$. 
Proof. (A) $\Rightarrow$ (B) Suppose $D \subseteq \mathbf{P}$ is dense in $\mathbf{P}$ and $\exists q \in \mathbf{Q}$ such that $\neg \exists p \in$ $D p$ and $q$ are compatible in $\mathbf{Q}$. Then for any $G, \mathbf{Q}$-generic over $M$ with $q \in G$, $G \cap \mathbf{P} \cap D=\varnothing$. If $p$ and $q$ are incompatible in $\mathbf{P}$ but $\exists r \in \mathbf{Q}, r<p$ and $r<q$, then any $G$, Q-generic with $r \in G$, has the property that $G \cap \mathbf{P}$ is not a $\mathbf{P}$ filter.

(B) $\Rightarrow$ (A) By the first clause $P \cap G$ meets every dense subset of $P$ in $M$ and by the second clause it is a $\mathbf{P}$ filter.

4. Iterated random reals. The main result of this section is the consistency of $\mathrm{B}(c)+\mathrm{B}(m)+\neg \mathrm{A}(c)+\neg \mathrm{A}(m)$. Let $\mathrm{B}$ be the partial order for iteratively adding $\omega_{2}$ random reals with finite support. That means that $p \in B$ iff $p \in M^{\omega_{2}}$ and $\forall \alpha<\omega_{2} p\left\lceil\alpha \mid\right.$ " $p(\alpha)$ is a nonzero element of the measure algebra of $2^{\omega}$ in $M\left[G_{\alpha}\right]$ " and $\operatorname{supp}(p)$ is finite. By c.c.c. it is clear that $M\left[G_{\omega_{2}}\right] \vDash " \mathrm{~B}(m)$ ". For any $\alpha<\omega_{2}$ let $z_{\alpha} \in 2^{\omega}$ be defined by $z_{\alpha}(n)=x_{\alpha+n}(0)$ where $x_{\alpha}$ is the $\alpha$ th random real added by $B$. Since supports are finite it is easily checked that $z_{\alpha}$ avoids every nowhere dense set coded in $M\left[G_{\alpha}\right]$ and therefore again by c.c.c. $M\left[G_{\omega_{2}}\right]$ F"B(c)".

Thus it remains to show that $M\left[G_{\omega_{2}}\right] \Vdash$ " $\neg \mathrm{A}(c)+\neg \mathrm{A}(m)$ ".

LEMMA 4.1. $\forall q \in \mathbf{B} \exists n<\omega \exists p \leqslant q$ such that

$$
\forall \alpha<\omega_{2} p \nmid \alpha \mid \vdash " \mu(p(\alpha))>1 /(n+1) " .
$$

Proof. This is easily proved by induction on $\max (\operatorname{supp}(q))$ by adding to the induction hypothesis $\max (\operatorname{supp}(q))=\max (\operatorname{supp}(p))$.

Lemma 4.2. Suppose $n<\omega, F \in\left[\omega_{2}\right]^{<\omega}$, and $\Vdash$ " $\tau \in \omega$ "; then $\exists q \in \mathbf{B} \exists N<\omega$

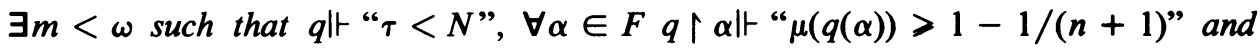
$\forall \alpha<\omega_{2} q \uparrow \alpha \Vdash$ " $\mu(q(\alpha))>1 /(m+1)$ ".

Proof. The proof is by induction on $(\min (F),|F|)($ where $\min (F)$ is the least element of $F$ ) over all possible ground models.

Case 1. $\min (F)=\alpha \neq 0$. In $M\left[G_{\alpha}\right]$ the lemma is true (with $M\left[G_{\alpha}\right]$ the ground model), so we can find $q \uparrow \alpha, H \in\left[\omega_{2}-\alpha+1\right]^{<\omega}, N<\omega, m<\omega$, and a term $\tilde{q}$ so that $q \uparrow \alpha \Vdash\left[\tilde{q} \mid\right.$ " $\tau<N$ ", $H=\operatorname{supp}(\tilde{q}), \forall \beta \alpha<\beta<\omega_{2}, \tilde{q} \uparrow[\alpha, \beta) \Vdash “ \mu(\tilde{q}(\beta))>$ $1 /(m+1)$ " and $\forall \beta \in F \tilde{q} \uparrow[\alpha, \beta) \Vdash$ " $\mu(\tilde{q}(\beta))>1-1 /(n+1)$ "]. Thus using $H$ we can define $q \in \mathbf{B}$ with the required properties.

Case 2. $\min (F)=0$. In $M\left[G_{0}\right]$ the lemma is true for $\hat{F}=F-\{0\}$, so choose a sequence in $M\left(q_{k}, H_{k}, \tilde{q}_{k}, N_{k}, m_{k}\right)$ for $k<\omega$ so that $\left\{q_{k}: k<\omega\right\}$ is a maximal family of incompatible elements of the measure algebra on $2^{\omega}$ and for all $k<\omega$, $H_{k} \in\left[\omega_{2}-\{0\}\right]^{<\omega}, N_{k}, m_{k}<\omega$, and

$$
\begin{aligned}
& q_{k} \Vdash\left[\operatorname{supp}\left(\tilde{q}_{k}\right)\right.=H_{k}, \forall \alpha \in \hat{F}, \tilde{q}_{k} \uparrow[1, \alpha) \Vdash “ \mu\left(\tilde{q}_{k}(\alpha)\right)>1-1 /(n+1) ”, \\
&\left.\forall \alpha \tilde{q}_{k} \uparrow[1, \alpha) \Vdash “ \mu\left(\tilde{q}_{k}(\alpha)\right)>1 /\left(m_{k}+1\right) ”, \text { and } q_{k} \Vdash “ \tau<N_{k} "\right]
\end{aligned}
$$

Choose $l<\omega$ so that $\Sigma_{i<l} \mu\left(q_{i}\right) \geqslant 1-1 / n$. Let $N=\sup \left\{N_{k}: k<l\right\}, q(0)=$ $\cup_{i<l} q_{i}, m=\sup \left\{m_{k}: k<l\right\}$, and for $\alpha>0$ let $q(\alpha)=q_{i}(\alpha)$ if $q_{i}$. Note that $\operatorname{supp}(q) \subseteq \cup\left\{H_{i}: i<l\right\}$ and therefore $q \in \mathbf{B}$ and is as required.

REMARK. All that is used to prove Lemmas 4.1 and 4.2 is that the measure algebra is a Boolean algebra with a finitely additive strictly positive measure. For example, the Boolean algebra of clopen subsets of $2^{\omega}$ would do as well. 
We show first that no $f \in \omega^{\omega} \cap M\left[G_{\omega_{2}}\right]$ eventually dominates every element of

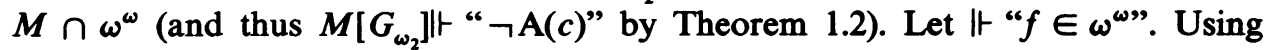
Lemma 4.2 construct a sequence $p_{n} \in \mathrm{B}$ for $n<\omega$ and $g \in \omega^{\omega}$ so that $\forall n<\omega$ $\operatorname{supp}\left(p_{n+1}\right) \supseteq \operatorname{supp}\left(p_{n}\right), \forall \alpha \in \operatorname{supp}\left(p_{n}\right) p_{n+1}\left\lceil\alpha \mid{ }^{\prime} " \mu\left(p_{n+1}(\alpha)\right)>1-1 /(n+1)\right.$ ", and $p_{n+1} \Vdash t$ " $f(n)<g(n)$ ".

Claim. It " $\exists{ }^{\infty} n f(n)<g(n)$ ".

Proof. Given any $q$ and $m<\omega$, by Lemma 4.1, $\exists p<q \quad \exists n>m \forall \alpha<\omega_{2}$ $p \nmid \alpha \mid \vdash$ “ $\mu(p(\alpha))>1 / n$ ", and $\operatorname{supp}(p) \cap \cup\left\{\operatorname{supp}\left(p_{k}\right): k<\omega\right\} \subseteq \operatorname{supp}\left(p_{n}\right)$. But then $p_{n+1}$ is compatible with $p$. To see this let $r(\alpha)$ be a term for $p_{n+1}(\alpha) \cap q(\alpha)$; then show by induction on $\alpha$ that $r \uparrow \alpha \in \mathbf{B}^{\alpha}$.

Case 1. If $\alpha \notin \operatorname{supp}(p) \cup \operatorname{supp}\left(p_{n+1}\right)$ then $r(\alpha)=2^{\omega}$.

Case 2. If $\alpha \in \operatorname{supp}(p)-\operatorname{supp}\left(p_{n+1}\right)$ then $r(\alpha)=p(\alpha)$.

Case 3. If $\alpha \in \operatorname{supp}\left(p_{n+1}\right)-\operatorname{supp}(p)$ then $r(\alpha)=p_{n+1}(\alpha)$.

Case 4. If $\alpha \in \operatorname{supp}(p) \cap \operatorname{supp}\left(p_{n+1}\right)$ then by choice of $n, \alpha \in \operatorname{supp}\left(p_{n}\right)$ and thus $p_{n+1} \uparrow \alpha \mid$ “ $\mu\left(p_{n+1}(\alpha)\right)>1-1 /(n+1)$ " and therefore

$$
r|\alpha| \vdash \text { “ } \mu\left(p_{n+1}(\alpha) \cap p(\alpha)\right)>0 " .
$$

To show $\neg \mathrm{A}(m)$ we need the following lemma.

LEMMA 4.3. If $\Vdash$ " $f \in \omega^{\omega}$ " then $\exists g \in \omega^{\omega} \exists p_{n} \in \mathbf{B}$ for $n<\omega$ such that $p_{n} \Vdash$ " $f(n)$ $<g(n)$ " and

$$
\forall q \in \mathbf{B} \exists n \forall k>n\left(q \cap \cap\left\{p_{l}: n<l<k\right\}\right) \in \mathbf{B} .
$$

Proof. Construct $p_{n} \in \mathrm{B}$ with increasing supports, $k_{n}<\omega$, and $g(n)$ by induction on $n<\omega$. Let $p_{0} \Vdash$ " $f(0)<g(0)$ " and $\forall \alpha<\omega_{2} p_{0} \uparrow \alpha \Vdash$ " $\mu\left(p_{0}(\alpha)\right)>2 / k_{0}$ ". Use Lemma 4.2 to find $p_{n+1}$ and $k_{n+1}>2^{k_{n}+1}$ so that

$$
\begin{aligned}
& \forall \alpha p_{n+1} \uparrow \alpha \text { |t “ } \mu\left(p_{n+1}(\alpha)\right)>2 / k_{n+1} \text { ", } \\
& \forall \alpha \in \operatorname{supp}\left(p_{n}\right) \quad p_{n+1} \uparrow \alpha \mid t c \mu\left(p_{n+1}(\alpha)\right)>1-1 / k_{n+1} \text { ", }
\end{aligned}
$$

and $p_{n+1} \Vdash$ " $f(n+1)<g(n+1)$ ". For any pair $n<k$ and $\alpha<\omega_{2}$ let $r(\alpha)$ be a term for the intersection $\cap\left\{p_{l}(\alpha): n<l<k\right\}$. If $\alpha \in \operatorname{supp}\left(p_{n}\right)$ then

$$
\begin{gathered}
r|\alpha| \vdash " \mu\left(2^{\omega}-r(\alpha)\right) \leqslant \sum_{n<l<k} \mu\left(2^{\omega}-p_{l}\right)<\sum_{n<l<k} \frac{1}{k_{l}}<\frac{1}{k_{n}} \\
\text { and therefore } \mu(r(\alpha))>\left(1-\frac{1}{k_{n}}\right) " .
\end{gathered}
$$

If $\alpha \in \operatorname{supp}\left(p_{i+1}\right)-\operatorname{supp}\left(p_{i}\right)$ for some $i, n<i<k-1$, then

$$
r(\alpha)=\cap\left\{p_{j}(\alpha): i+1<j<k\right\}
$$

and thus

$$
\begin{aligned}
& r \uparrow \alpha \mid \vdash " \mu\left(2^{\omega}-r(\alpha)\right)<\mu\left(2^{\omega}-p_{i+1}(\alpha)\right)+\sum_{i+1<j<k} \mu\left(2^{\omega}-p_{j}(\alpha)\right) \\
& \quad<\left(1-\frac{1}{k_{i+1}}\right)+\frac{1}{k_{i+1}}=1 \text { and thus } \mu(r(\alpha))>0 " .
\end{aligned}
$$


Given any $q$ choose $p \leqslant q$ and $n$ sufficiently large so that $\forall \alpha p \uparrow \alpha \Vdash$ “ $\mu(p(\alpha))>$ $1 / k_{n} "$ and $\operatorname{supp}(p) \cap \cup\left\{\operatorname{supp}\left(p_{i}\right): i<\omega\right\} \subseteq \operatorname{supp}\left(p_{n}\right)$, these exist by Lemma 4.1. By an argument similar to the proof of the claim, $p$ and $r$ are compatible and thus $q$ and $r$ are compatible.

This lemma implies, by a density argument, that for every real (element of $\omega^{\omega}$ ) in the extension there is a real in the ground model which dominates it on arbitrarily large blocks of consecutive integers. Suppose $\mathbb{F}^{\text {" }} G \subseteq 2^{\omega}$ is an open set of measure $<\frac{1}{4}$ ". By Lemma 4.3 we may find $n_{k}<n_{k+1}<\omega$ with $n_{0}=0$ and $p_{k}$ for $k<\omega$ such that

$$
p_{k} \Vdash " \forall s \in 2^{<n_{k}} \mu\left(G \cap[s]-\bigcup\left\{[t]: t \in 2^{n_{k+1}} \text { and }[t] \subseteq G\right\}\right) \leqslant \frac{1}{2^{2 n_{k}+3}} \text { ", }
$$

and $\forall q \in \mathbf{B} \exists n \forall k>n, \cap\left\{p_{l}: n<l<k\right\}$ and $q$ are compatible. Choose disjoint $F_{k} \subseteq\left\{n_{i}: i<\omega\right\}$ with $\left|F_{k}\right|=k$, and consider $H=\left\{X \in 2^{\omega}: \exists^{\infty} n \forall i \in F_{n}, x(i)\right.$ $=1\}$. $H$ is a measure zero set coded in $M$. For any $G$, B-generic over $M$, let $Y=\left\{n_{i}: p_{i} \in G\right\}$ and let $C=\left\{x \in 2^{\omega}: \forall n \in Y x(n)=1\right\}$. A density argument shows that $C \subseteq H$. As in the proof of Theorem 1.6, $C \nsubseteq G$ and therefore $H \nsubseteq G$. Since $G$ was arbitrary $\neg \mathrm{A}(m)$ holds in $M\left[G_{\omega_{2}}\right]$.

5. Eventually different reals. In this section we show that $\mathrm{B}(c)+\mathrm{U}(c)+\neg \mathrm{B}(m)$ $+\neg \mathrm{A}(c)$ is consistent. Let $\mathbf{E}$ be the following partial order. $\mathbf{E}=\left\{(s, G): s \in \omega^{<\omega}\right.$ and $\left.G \in\left[\omega^{\omega}\right]^{<\omega}\right\}$ and $(s, G) \leqslant(t, H)$ iff $s \supseteq t, G \supseteq H$, and $\forall i$ if $|s|<i<|t|$ then $\forall g \in H, t(i) \neq g(i)$. A density argument shows that if

$$
f=\cup\{s:(s, H) \in G\}
$$

for any $G$ E-generic over $M$ then $\forall g \in \omega^{\omega} \cap M, \forall^{\infty} n g(n) \neq f(n)$. Let $\mathbf{P}_{\alpha}$ denote the $\alpha$ iteration of $\mathbf{E}$ with finite support (if $p \in \mathbf{P}_{\alpha}$ then $\forall \beta<\alpha p \uparrow \beta$ $\Vdash^{\prime}$ " $p(\beta) \in \mathbf{E}^{\left.M\left[G_{\beta}\right] ”\right) . ~ A s ~ i n ~} \S 4$ finite support means that Cohen reals are added, and also eventually different reals are added. By Theorem $1.3 M\left[G_{\omega_{2}}\right] \Vdash$ "B(c) $+\mathrm{U}(c)$ " for any $G_{\omega_{2}} \mathbf{P}_{\omega_{2}}$-generic over $M$.

We claim that no real in $M\left[G_{\omega_{2}}\right]$ eventually dominates the reals in $M$ (so by Theorem $1.2 \neg \mathrm{A}(c)$ holds in $\left.M\left[G_{\omega_{2}}\right]\right)$. For clarity we first show this for a single step, that is, $\forall G$ E-generic over $M, M[G] \Vdash{ }^{\prime \prime} \forall f \in \omega^{\omega}, \exists g \in \omega^{\omega} \cap M, \exists^{\infty} n f(n)<$ $g(n)$ ".

Lemma 5.1. Suppose $\Vdash$ “ $\tau \in M$ ", $s \in \omega^{<\omega}$, and $n<\omega$ then $\exists H \in[M]^{<\omega} \forall G \subseteq$ $\omega^{\omega}$ if $|G| \leqslant n$ then $\exists p \leqslant(s, G), p \Vdash$ “ $\tau \in H$ ".

Proof. $\forall H \in[M]^{<\omega}$ let $G_{H}=\left\{\left(g_{0}, \ldots, g_{n-1}\right) \in\left(\omega^{\omega}\right)^{n}: \exists p<\right.$ $\left(s,\left\{g_{0}, \ldots, g_{n-1}\right\}\right)$ such that $p \Vdash$ " $\left.\tau \in H^{\prime}\right\}$. Note $\left\{G_{H}: H \in[M]^{<\omega}\right\}$ is a cover of $\left(\omega^{\omega}\right)^{n}$. Give $\omega$ the cofinite topology, i.e. $U \subseteq \omega$ is open iff $U$ is cofinite, and give $\omega^{\omega}$ and $\left(\omega^{\omega}\right)^{n}$ the product topology. Then by Tychonoff's theorem $\left(\omega^{\omega}\right)^{n}$ is compact. For any $\left(g_{0}, \ldots, g_{n-1}\right) \in G_{H}$ if $p=(t, H) \leqslant\left(s,\left\{g_{0}, \ldots, g_{n-1}\right\}\right)$ and $p$ | " $\tau \in$ $H^{\prime \prime}$, then let $U=\left\{\left(g_{1}^{\prime}, \ldots, g_{n}^{\prime}\right): \forall i\right.$ if $|s| \leqslant i<|t|$ then $\left.\forall k<n t(i) \neq g_{k}^{\prime}(i)\right\}$. Note that $U$ is a basic open set and $g \in U \subseteq G_{H}$ since $p<\left(s,\left\{g_{0}^{\prime}, \ldots, g_{n-1}^{\prime}\right\}\right)$ for any $\left(g_{0}^{\prime}, \ldots, g_{n-1}^{\prime}\right) \in U$. Since $G_{H} \cup G_{K} \subseteq G_{H \cup K}, \exists H \in[M]^{<\omega} G_{H}=\left(\omega^{\omega}\right)^{n}$. 
Let $\left(s_{n}, k_{n}\right)$ for $n<\omega$ list with infinitely many repetitions $\omega^{<\omega} \times \omega$. Let $\Vdash$ " $f \in$ $\omega^{\omega}$ " and using Lemma 5.1 find $g \in \omega^{\omega}$ so that $\forall n \forall G \in\left[\omega^{\omega}\right]^{k_{n}} \exists p<\left(s_{n}, G\right)$ $p \Vdash$ " $f(n)<g(n)$ ". Now suppose $\exists n \exists p p \Vdash$ " $\forall m>n f(m)>g(m)$ "; then $\exists m>n$ such that $\left(s_{m}, G\right)=p$ and $|G|=k_{m}$, but then $\exists q \leqslant p q \Vdash$ " $f(m)<g(m)$ ", which is a contradiction. Thus by genericity, $\exists^{\infty} n f(n)<g(n)$.

Next we show that no random reals are added by one step.

Lemma 5.2. Suppose $\Vdash$ " $\tau<N$ " where $N<\omega$ and $s \in \omega^{<\omega}$; then $\exists n<N$ such that $\forall H \in\left[\omega^{\omega}\right]^{<\omega} \exists p \leqslant(s, H) p \Vdash$ " $\tau=n$ ".

Proof. If not, then $\forall i<N \exists H_{i}\left(s, H_{i}\right) \Vdash$ “ $\tau \neq i$ ”. But $\left(s, \cup\left\{H_{i}: i<N\right\}\right) \Vdash$ “ $\forall i$ $<N, \tau \neq i$, which is a contradiction.

For any $x \in 2^{\omega} \cap M[G]$, by employing an argument similar to the one following Lemma 5.1 but instead using Lemma 5.2, there exists $g \in M$ such that $g: \omega \rightarrow 2^{<\omega}$ and $\exists^{\infty} n x \uparrow n=g(n)$. Note that $H=\left\{y \in 2^{\omega}: \exists^{\infty} n\right.$ y $\left.n=g(n)\right\}$ has measure zero (assuming $\forall n g(n) \in 2^{n}$ ) so $x$ is not random over $M$.

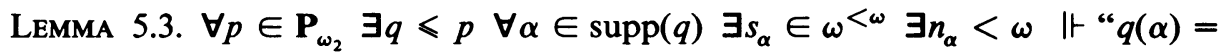
$\left(s_{\alpha}, G\right)$ for some $G \in\left[\omega^{\omega}\right]^{n_{\alpha}}$.

Proof. The proof is by induction on $\max (\operatorname{supp}(p))$.

Call such $q$ as above canonical and from now on we assume all conditons in $\mathbf{P}_{\omega_{2}}$ are canonical (since the canonical ones are dense).

LEMMA 5.4. Suppose $\mathbb{t}$ " $\tau \in M$ " and given $F \in\left[\omega_{2}\right]^{<\omega}$ and $n_{\alpha}<\omega$ and $s_{\alpha} \in \omega^{<\omega}$ for every $\alpha \in F$, then $\exists H \in[M]^{<\omega}$ such that $\forall q \in \mathbf{P}_{\omega_{2}}$ if $\operatorname{supp}(q)=F$ and $\forall \alpha \in$ $F, n_{\alpha}^{q}=n_{\alpha}$ and $s_{\alpha}^{q}=s_{\alpha}$ then $\exists p \leqslant q p \Vdash$ " $\tau \in H$ ".

Proof. The induction is on $(\min (F),|F|)$ as in Lemma 4.2 and the case $0 \neq \min (F)$ is proved similarly. If $0=\min (F)$ then since the lemma is true with $M\left[G_{0}\right]$ as the ground model let $\bar{H}$ be a term in the forcing language of $\mathbf{P}$ such that

$\Vdash$ " $\bar{H} \in[M]^{<\omega}$ and $\forall q \in \mathbf{P}^{\left[1, \omega_{2}\right)}$ if $\operatorname{supp}(q)=F-\{0\}$

and $\forall \alpha \in F-\{0\}, n_{\alpha}^{q}=n_{\alpha}$ and $s_{\alpha}^{q}=s_{\alpha}$ then $\exists p<q, p \Vdash \tau \in \bar{H}$ ".

Now use Lemma 5.3 to find $H$ and take care of $s_{0}$ and $n_{0}$.

Lemma 5.5. Suppose $\Vdash$ " $\tau<N$ " where $N<\omega$ and given $F \in\left[\omega_{2}\right]<\omega$ and $n_{\alpha}<\omega$ and $s_{\alpha} \in \omega^{<\omega}$ for every $\alpha \in F$, then $\exists n<N$ such that $\forall q \in \mathbf{P}_{\omega_{2}}$ with $\operatorname{supp}(q)=F$ and $\forall \alpha \in F, n_{\alpha}=n_{\alpha}^{q}$ and $s_{\alpha}=s_{\alpha}^{q} \exists p \leqslant q p \Vdash$ “ $\tau=n$ ".

Proof. The proof of this lemma is similar to Lemma 5.4 using Lemma 5.2 instead of Lemma 5.1.

It is now easy to see that $\forall \alpha<\omega_{1} \forall G_{\alpha} P_{\alpha}$-generic over $M \forall f \in \omega^{\omega} \cap M\left[G_{\alpha}\right]$, $\exists g \in \omega^{\omega} \cap M, \exists^{\infty} n f(n)<g(n)$ and

$$
2^{\omega} \cap M\left[G_{\alpha}\right]=\cup\{G: \mu(G)=0 \text { and } G \text { is coded in } M\} .
$$

The proofs are similar to before, just list in an $\omega$ sequence all the sets $\left(F,\left(\left(s_{\alpha}, n_{\alpha}\right): \alpha \in F\right)\right)$ for $F \in[\alpha]^{<\omega}$. The remaining lemmas will reduce us to this case. The technique is similar to that of the proof of Lemmas 28 through 30 of [M2]. 
Lemma 5.6. Suppose $M \subseteq N$ are transitive models of $Z F C$. Then if $G$ is $\mathbf{E}^{N}$-generic over $N$ then $G \cap \mathbf{E}^{M}$ is $\mathbf{E}^{M}$-generic over $M$.

Proof. The proof is exactly the same as Lemma 5.2 of [T]. Namely if $M F^{\text {“ }} A \subseteq$ $\mathbf{E}$ is a maximal antichain" then $A$ is a maximal antichain in $\mathbf{E}$ by $\Pi_{1}^{1}$ absoluteness.

Call a partial order $\mathbf{P}$ absolute just in case it is definable (possibly with parameters in $M$ ) and given any $N \supseteq M$, a transitive model of ZFC if $G$ is $\mathbf{P}^{N}$-generic over $N$, then $G \cap \mathbf{P}^{M}$ is $\mathbf{P}^{M}$-generic over $M$. Suppose $\mathbf{P}_{\alpha}$ for $\alpha<\gamma$ is a finite support iteration of absolute partial orders over $M$, i.e. $\mathbf{P}_{\alpha+1}=\mathbf{P}_{\alpha} * \mathbf{P}^{\alpha}$ where $\mathbf{P}^{\alpha}$ is some name for an absolute partial order in $M\left[G_{\alpha}\right]$. Given $X \subseteq \gamma(X \in M)$ define the iteration $\mathbf{P}_{\alpha}^{*}$ for $\alpha \leqslant \gamma$ as follows:

For $\alpha \notin X$ let $\mathbf{P}_{\alpha+1}^{*}=\mathbf{P}_{\alpha}^{*} * 1$ (where 1 is the one element order).

For $\alpha \in X$ let $\mathbf{P}_{\alpha+1}^{*}=\mathbf{P}_{\alpha}^{*} *\left(\mathbf{P}^{\alpha}\right)^{M\left[G_{\alpha}^{*}\right]}$ where $G_{\alpha}^{*}$ is $\mathbf{P}_{\alpha}^{*}$-generic over $M$.

For $G_{\alpha} \mathbf{P}_{\alpha}$-generic over $M$ define $G_{\alpha}^{*}=G_{\alpha} \cap \mathbf{P}_{\alpha}^{*}$.

Lemma 5.7. $\forall \alpha \leqslant \gamma$ if $G_{\alpha}$ is $\mathbf{P}_{\alpha}$-generic over $M$ then $G_{\alpha}^{*}$ is $\mathbf{P}_{\alpha}^{*}$-generic over $M$.

Proof. The proof is by induction on $\alpha$. For the successor case $\alpha+1$ if $\alpha \notin X$ then $\mathrm{P}_{\alpha+1}^{*}$ is isomorphic to $\mathrm{P}_{\alpha}^{*}$. Now suppose $\alpha \in X$ then $G_{\alpha+1}=G_{\alpha} \times G^{\alpha}$ where $G^{\alpha}$ is $\left(\mathbf{P}^{\alpha}\right)^{M\left[G_{\alpha}\right]}$-generic over $M\left[G_{\alpha}\right]$. By absoluteness $G^{* \alpha}=G^{\alpha} \cap\left(P^{\alpha}\right)^{M\left[G_{\alpha}^{*}\right]}$ is $\left(\mathrm{P}^{\alpha}\right)^{M\left[G_{\alpha}^{*}\right]}$-generic over $M\left[G_{\alpha}^{*}\right]$. So $G_{\alpha+1}^{*}=G_{\alpha}^{*} \times G^{* \alpha}$ is $\mathbf{P}_{\alpha+1}^{*}$-generic over $M$ by the product lemma (see [So]). Now suppose $\alpha$ is a limit ordinal. We will use the lemma in §3. Suppose $D \subseteq \mathbf{P}_{\alpha}^{*}$ is dense; then we need to show $E=\left\{q \in \mathbf{P}_{\alpha}: \exists p \in D\right.$ $q<p\}$ is dense in $\mathbf{P}_{\alpha}$. Given $q \in \mathbf{P}_{\alpha}$ choose $\beta$ such that $\operatorname{supp}(q) \subseteq \beta$. Note that $\{p \uparrow \beta: p \in D\}$ is dense in $\mathbf{P}_{\beta}^{*}$, so $\exists p \in D p \uparrow \beta$ and $q \uparrow \beta$ are compatible in $\mathbf{P}_{\beta}$; therefore $p$ and $q$ are compatible in $\mathbf{P}_{\alpha}$. Note that the fact, that two conditions in $\mathbf{P}_{\alpha}^{*}$ are compatible in $\mathbf{P}_{\alpha}^{*}$ iff they are compatible in $\mathbf{P}_{\alpha}$, is easy since $\alpha$ is a limit ordinal.

LeMma 5.8. If " $\tau \subseteq \omega$ " then $\exists X \subseteq \omega_{2}$ countable, $X \in M$ and $\forall G \mathbf{P}_{\omega_{2}}$-generic over $M \tau^{G} \in M\left[G^{*}\right]$.

Proof. This is easy using c.c.c.

Thus we conclude that

$$
\begin{array}{r}
M\left[G_{\omega_{2}}\right] \cap \omega^{\omega}=\bigcup\left\{M\left[H_{\alpha}\right] \cap \omega^{\omega}: \alpha<\omega_{1}, H_{\alpha} \in M\left[G_{\omega_{2}}\right],\right. \\
\text { and } \left.H_{\alpha} \text { is } \mathbf{P}_{\alpha} \text {-generic over } M\right\} .
\end{array}
$$

Therefore we have shown

TheOREM 5.9. $\mathrm{B}(c)+\mathrm{U}(c)+\neg \mathrm{B}(m)+\neg \mathrm{A}(c)$ is consistent with $Z F C$.

Let $\mathrm{D}$ be the dominating partial order, $\mathrm{D}=\left\{(n, f): n<\omega, f \in \omega^{\omega}\right\}$ where $(n, f) \leqslant(m g)$ iff $n>m, f \uparrow m=g \uparrow m$, and $\forall i f(i)>g(i)$ (see [H]). Note that Lemma 5.2 is also true for $\mathbf{D}$. Hence by similar arguments no random reals are added by a finite support iteration of $\mathbf{D}$. This shows

THEOREM 5.10. $\mathrm{A}(c)+\neg \mathrm{B}(m)$ is consistent with $Z F C$. 
REMARK. Kunen first proved Theorem 5.10 using the $\aleph_{1}$ precalibre of $\mathbf{D}$ and the fact that the measure algebra on $2^{\omega}$ (assuming $\mathrm{CH}$ ) does not have $\aleph_{1}$ precalibre.

6. Mathias reals. Let $\mathbf{P}=\left\{(s, A): s \in[\omega]^{<\omega}, A \in[\omega]^{\omega}\right.$, and $\left.\sup (s)<\inf (A)\right\}$ where $(s, A) \leqslant(t, B)$ iff $s \supseteq t$ and $(s-t) \cup A \subseteq B$ (see [Ma]).

Lemma 6.1. $\forall G$ P-generic over $M, M[G] \vDash^{*} M \cap 2^{\omega}$ is meager and has measure zero".

Proof. Let $Z=\cup\{s: \exists A(s, A) \in G\}$ and note that a density argument shows that $\forall X \in P(\omega) \cap M \exists n<\omega Z-n \subseteq X$ or $(Z-n) \cap X=\varnothing$. But for any infinite $Q \subseteq \omega, \cup_{i=0,1}\left\{X \in 2^{\omega}: \forall n \in Q x(n)=i\right\}$ is a closed set of measure zero (hence nowhere dense).

The following statement is due to Mathias (see [Ma]).

$$
\forall(s, A) \forall \theta \exists B \subseteq A(s, B) \Vdash \text { “ } \theta \text { ” or }(s, B) \Vdash \text { “ } \neg \theta \text { ”. }
$$

Lemma 6.2. Suppose $\Vdash$ “ $\tau \in 2^{\omega}$ "; then $\exists B=\left\{b_{n}: n<\omega\right\} \subseteq \omega$ so that $\forall m<\omega$ $\forall t \subseteq\left\{b_{n}: n \leqslant m\right\} \exists r \in 2^{b_{m}}$ so that

$$
\left(t, B-\left(b_{m}+1\right)\right) \Vdash \text { “ } \tau \uparrow b_{m}=r " .
$$

Proof. Construct $B_{n} \in[\omega]^{\omega}$ and $\left\{b_{m}: m<n\right\}$ by induction on $n$. Let $B_{0}=\omega$ and choose $b_{n} \in B_{n}-\left(b_{n-1}+1\right)$ arbitrarily. By repeated applications of $*$ obtain $B_{n+1} \subseteq B_{n}-\left(b_{n}+1\right)$ so that $\forall t \subseteq\left\{b_{m}: m<n\right\} \exists r \in 2^{b_{n}}$ so that $\left(t, B_{n+1}\right) \Vdash{ }^{~ "} \tau \uparrow$ $b_{n}=r$ '.

For clarity we show first that no real in $M[G]$, for $G \mathbf{P}$-generic over $M$, is Cohen or random over $M$. So let $\Vdash t$ “ $\tau \in 2^{\omega}$ ". By refining $B=\left\{b_{n}: n<\omega\right\}$ from Lemma 6.2 we can assume that $\forall n<\omega, b_{n} \geqslant 2^{2^{n+1}}$. Define $T=\left\{t \in 2^{<\omega}: \exists q<(\varnothing, B)\right.$ $\exists n<\omega q \Vdash$ “ “ $\tau \mid n=t$ ” $\}$. By Lemma 6.2, $\forall n<\omega\left|T \cap 2^{b_{n}}\right|<2^{n+1}$ and thus $\mid T \cap$ $2^{2^{n+1}} \mid<2^{n+1}$. Therefore $C=\left\{x \in 2^{\omega}: \forall n x \uparrow n \in T\right\}$ is a closed set of measure zero (hence nowhere dense) and $(\varnothing, B) \vdash$ " $\tau \in C$ ".

Now let $\mathbf{P}_{\omega_{2}}$ be the $\omega_{2}$ iteration of $\mathbf{P}$ with countable support. Let $X=\{x \in$ $\left.\omega^{\omega}: \forall n x(n)<2^{n^{3}}\right\}$. We claim that $\forall y \in M\left[G_{\omega_{2}}\right] \cap X \exists\left\langle F_{n}: n<\omega\right\rangle \in M$ such that $\forall n\left(\left|F_{n}\right| \leqslant 2^{n^{2}}\right.$ and $\left.y(n) \in F_{n}\right)$. Define $\theta: X \rightarrow 2^{\omega}$ by $\theta(x)=x(0) \frown x(1) \frown$ $x(2) \frown \ldots$, where we identify $2^{n^{3}}$ with sequences of 0 's and l's of length $n^{3}$. If $C=\left\{x \in X: \forall n x(n) \in F_{n}\right\}$ then

$$
\mu(\theta(C))=\lim _{n \rightarrow \infty} \frac{\left|F_{n}\right|}{2^{n^{3}}}=\lim _{n \rightarrow \infty} \frac{1}{2^{n}}=0 .
$$

Thus it remains only to prove this claim. Define $(s, X)<_{n}(t, Y)$ for $n<\omega$ iff $s=t, X \subseteq Y$, and the first $n$ elements of $Y$ are still in $X$. Using $*$ it is possible to show that if $p \Vdash t<<N$ " where $N<\omega$ then $\forall n<\omega \exists q<_{n} p \exists H|H|<2^{n}$ $q$ |t “ $\tau \in H$ ". For $F \in\left[\omega_{2}\right]^{<\omega}$ define for $p, q \in \mathbf{P}^{\omega_{2}}, p<{ }_{n}^{F} q$ iff $p<q$, and $\forall \alpha \in F$ $p \nmid \alpha \mid t$ " $p(\alpha) \leqslant n q(\alpha)$ ". Then it can be shown that $\forall p \in \mathbf{P}_{\omega_{2}} \forall n<\omega \forall F \in\left[\omega_{2}\right]^{n}$ $\exists q<_{n}^{F} p \exists H|H| \leqslant 2^{n^{2}} q \Vdash$ “ $\tau \in H$ ". A fusion argument now finishes the proof of the claim.

REMARK. In fact we have shown that

$$
M\left[G_{\omega_{2}}\right] \cap 2^{\omega}=\cup\{C: C \text { closed measure zero and coded in } M\} \text {. }
$$


Thus $U(m)$ does not imply that the union of less than $\left|2^{\omega}\right|$ closed sets of measure zero has measure zero.

7. Infinitely often equal reals. In this section we show that $\mathrm{U}(m)+\neg \mathrm{U}(c)+$ $\neg \mathrm{B}(c), \mathrm{U}(m)+\mathrm{B}(m)+\neg \mathrm{A}(m)+\neg \mathrm{B}(c)$, and $\neg \mathrm{U}(m)+\neg \mathrm{U}(c)$ are each consistent with ZFC. A Sacks real is obtained by forcing with perfect (every node has incompatible extensions) subtrees of $2^{<\omega}$ (see [Sa]). A Silver real is obtained by forcing with condition of the form $p: D \rightarrow 2$ where $D \subseteq \omega$ is coinfinite (see [G]). It is an unpublished result of Sacks (see also $[\mathbf{J}]$ ) that when one adds $\omega_{2}$ (or more) Sacks reals side by side with countable support then $\neg \mathrm{U}(m)+\neg \mathrm{U}(c)$ holds in the extension. The same is true when Silver reals are added.

Consider the partial order

$$
\mathbf{P}=\left\{s: D \rightarrow 2^{<\omega}: D \subseteq \omega \text { is coinfinite and } \forall n \in D, s(n) \in 2^{n}\right\}
$$

where $s \leqslant t \leftrightarrow s \supseteq t$. For any $G$ P-generic over $M$, define $f=\cup G$. An easy density argument shows that $\forall x \in M \cap 2^{\omega} \exists^{\infty} n x \uparrow n=f(n)$. Note that $\{x \in$ $\left.2^{\omega}: \exists^{\infty} n x \uparrow n=f(n)\right\}$ has measure zero for any $f$. It is also hard not to show that $2^{\omega} \cap M$ has strong measure zero (see Theorem 1.7). Next we show that $\forall h \in$ $M[G] \cap \omega^{\omega} \exists g \in M \cap \omega^{\omega} \forall n h(n)<g(n)$.

Lemma 7.1. Suppose $s \Vdash$ " $\tau<\omega$ " and $E \in[\omega-\operatorname{dom}(s)]<\omega$; then $\exists N<\omega \exists t<s$ $\operatorname{dom}(t) \cap E=\varnothing$ and $t \Vdash " \tau<N "$.

Proof. Let $\left\{r_{1}, \ldots, r_{k}\right\}=\left\{r: E \rightarrow 2^{<\omega}: \forall n \in E r(n) \in 2^{n}\right\}$. Successively extend $s>s_{1}>s_{2}>\cdots>s_{k}=t$ so that $\forall i E \cap \operatorname{dom}\left(s_{i}\right)=\varnothing$ and $s_{i} \cup r_{i} \Vdash$ " $\tau=$ $n_{i}$. Let $t=s_{k}$ and $N=\sup \left\{n_{i}: 1 \leqslant i \leqslant k\right\}+1$ and then $t \mid t$ " $\tau<N$ ".

Now suppose $s \Vdash$ “ $\tau \in \omega^{\omega}$ ". Construct $E_{n} \subsetneq E_{n+1}$ finite, $s_{n+1}<s_{n}<s_{0}=s$, and $g \in \omega^{\omega}$ such that $\operatorname{dom}\left(s_{n}\right) \cap E_{n}=\varnothing$ and $s_{n} \mathbb{t}$ " $\tau(n)<g(n)$ ". Then $t=$ $\cup\left\{s_{n}: n<\omega\right\}$ is in $\mathbf{P}$ since $\cup\left\{E_{n}: n<\omega\right\} \cap \operatorname{dom}(t)=\varnothing$, and $t \Vdash " \forall n \tau(n)<$ $g(n)$ ". Next we show that $M[G] k^{*} M \cap 2^{\omega}$ is not meager".

Lemma 7.2. Suppose $s \mid t$ " $C$ is nowhere dense in $2^{\omega "}, E \in[\omega-\operatorname{dom}(s)]^{<\omega}$, and $t \in 2^{<\omega}$. Then $\exists r \supset t \exists \bar{s}<s E \cap \operatorname{dom}(\bar{s})=\varnothing$ and $\bar{s} \mid$ " $[r] \cap C=\varnothing "$.

Proof. As before let $\left\{r_{i}: i<k\right\}=\left\{r: E \rightarrow 2^{<\omega}: \forall n \in E r(n) \in 2^{\omega}\right\}$. Now build $s_{i+1}<s_{i}<s$ for $i<k$ and $t_{i+1} \supseteq t_{i} \supseteq t$ such that $\operatorname{dom}\left(s_{i}\right) \cap E=\varnothing$ and $s_{i} \cup r_{i} \Vdash$ " $\left[t_{i}\right] \cap C=\varnothing$ ". This can be done since $s \Vdash$ " $C$ is nowhere dense". Then let $\bar{s}=s_{k-1}$ and $r=t_{k-1}$.

Now suppose $s \mid$ " “ $C \subseteq 2^{\omega}$ is nowhere dense". Let $2^{<\omega}=\left\{t_{n}: n<\omega\right\}$ and construct $s_{n+1}<s_{n}<s, E_{n+1} \supsetneq E_{n}$ finite, and $\bar{t}_{n} \supseteq t_{n}$ such that $s_{n} \Vdash$ " $C \cap \cap\left[\bar{t}_{n}\right]=$ $\varnothing$ " and $E_{n} \cap \operatorname{dom}\left(s_{n}\right)=\varnothing$. Then $\bar{s}=\cup\left\{s_{n}: n<\omega\right\}$ is in P since

$$
\bigcup\left\{E_{n}: n<\omega\right\} \cap \operatorname{dom}(\bar{s})=\varnothing
$$

and if $G=\cup\left\{\left[\bar{t}_{n}\right]: n<\omega\right\}$ then $G$ is open dense and $\bar{s} \mid$ " $G \cap C=\varnothing$ ". This shows that every meager set coded in $M[G]$ is covered by a meager set coded in $M$, so $M \cap 2^{\omega}$ is not meager in $M[G]$.

Next we are going to force with the side by side $\omega_{2}$ product of $\mathbf{P}$ with countable support to show that $\mathrm{U}(m)+\neg \mathrm{U}(c)+\neg \mathrm{B}(c)$ is consistent. For any set $X$ define 
${ }^{x} \mathbf{P}=\{p: X \rightarrow \mathbf{P}:\{x \in X: p(x) \neq \varnothing\}=\operatorname{supp}(p)$ is countable $\}$ where $p<q \leftrightarrow$ $\forall x \in X, p(x) \leqslant q(x)$. Since $|\mathbf{P}|=\omega_{1}$ a standard $\Delta$-system argument implies ${ }^{\omega_{2}} \mathbf{P}$ has the $\omega_{2}$ chain condition. Hence $\forall X \subseteq 2^{\omega}$ if $|X| \leqslant \omega_{1}$ and $X \in M\left[G_{\omega_{2}}\right]$ then $\exists \alpha<$ $\omega_{2} X \in M\left[G_{\alpha}\right]$. Note that $\forall x \in M\left[G_{\alpha}\right] \cap 2^{\omega} \forall n<\omega D=\left\{p \in \mathbf{P}^{M}: \exists m>n\right.$ $p(m)=x \uparrow m\}$ is dense in $\mathbf{P}$ and it is an element of $M\left[G_{\alpha}\right]$. It follows that $M\left[G_{\alpha}\right] \cap 2^{\omega}$ has measure zero in $M\left[G_{\omega_{2}}\right]$.

We claim $\forall f \in M\left[G_{\omega_{2}}\right] \cap \omega^{\omega}, \exists g \in \omega^{\omega} \cap M, \forall n<\omega, f(n)<g(n)$. This property (SD of $\S 1$ ) implies $\neg \mathrm{B}(c)$.

LemMA. 7.3. Given $p \in{ }^{\omega_{2}} \mathbf{P}, \tau$ such that $p \Vdash$ " $\tau<\omega$ ", $F \in\left[\omega_{2}\right]^{<\omega}$ and $E_{x} \in[\omega]^{<\omega}$ for all $x \in F$ such that $\forall x \in F \operatorname{dom}(p(x)) \cap E_{x}=\varnothing$, then $\exists N<\omega \quad \exists q<p$ $\forall x \in F \operatorname{dom}(q(x)) \cap E_{x}=\varnothing$ and $q \Vdash$ " $\tau<N$ ".

The proof is similar to Lemma 7.1 and is left to the reader.

To prove the claim suppose that $p \Vdash$ " $\tau \in \omega^{\omega}$ " and using Lemma 7.3 construct a sequence $p_{n+1} \leqslant p_{n} \leqslant p, E_{\alpha}^{n} \in[\omega]^{<\omega}$ for $\alpha<\omega_{2}$, and $g \in \omega^{\omega}$ such that $\forall \alpha E_{\alpha}^{n+1}$ $\supseteq E_{\alpha}^{n}$ and $E_{\alpha}^{n} \cap \operatorname{dom}\left(p_{n}(\alpha)\right)=\varnothing, p_{n} \Vdash$ “ $\tau(n)<g(n)$ ", for all but finitely many $\alpha$, $E_{n}^{\alpha}=\varnothing$, and $\forall \alpha \in \cup\left\{\operatorname{supp}\left(p_{n}\right): n<\omega\right\} \exists m \forall n>m E_{n+1}^{\alpha} \supsetneq E_{n}^{\alpha}$. This can be done by diagonalizing over the supports of the $p_{n}$ 's. Define $p(\alpha)=\cup\left\{p_{n}(\alpha): n<\right.$ $\omega\}$ for each $\alpha$; then we have that $p \in \omega_{2} \mathbf{P}$ and $p \Vdash$ " $\forall n \tau(n)<g(n)$ ". This proves the claim. To prove that $M \cap 2^{\omega}$ is not meager in $M\left[G_{\omega_{2}}\right]$ an argument analogous to Lemma 7.2 may be used.

Let $\delta$ be Silver forcing $(\mathcal{S}=\{p: D \rightarrow 2: D \subseteq \omega$ is coinfinite $\}) . \forall G$, $\delta$-generic over $M, M[G] \vDash^{\text {“ }} M \cap 2^{\omega}$ does not have measure zero". To see this note that Lemma 7.1 and the fusion argument following it can be improved in this case to show $\forall f \in M[G] \cap \omega^{\omega}, \exists g \in M, \forall n\left(f(n) \in g(n) \wedge|g(n)|<2^{n}\right)$. It is not hard (but it is messy) to show that $\forall X \subseteq 2^{\omega}$ if $\mu(X)=0$ then $\forall \varepsilon: \omega \rightarrow \mathbf{R}^{+} \exists C_{n} \subseteq 2^{\omega}$ clopen such that $\forall n<\omega \mu\left(C_{n}\right) \leqslant \varepsilon(n)$ and $X \subseteq \cup\left\{C_{n}: n<\omega\right\}$. Given $\left\langle C_{n}: n\right.$ $\langle\omega\rangle \in M[G]$ such that $\forall n C_{n}$ is clopen and $\mu\left(C_{n}\right)<1 / 2^{2 n+1}$. Since clopen sets can be coded by integers there exists $\left\langle\bigodot_{n}: n\langle\omega\rangle \in M\right.$ such that each $\bigodot_{n}$ consists of $\leqslant 2^{n}$ clopen sets of measure $\leqslant 1 / 2^{2 n+1}$ such that $\forall n C_{n} \in \mathcal{C}_{n}$. Note that $\mu\left(\cup\left\{\cup \mathcal{C}_{n}: n<\omega\right\}\right) \leqslant \frac{1}{2}$ and therefore it follows that every measure zero set coded in $M[G]$ is contained in a measure zero set coded in $M$.

A similar argument yields that for $G_{\omega_{2}}, \omega_{2} \delta$-generic over $M$, every measure zero set coded in $M\left[G_{\omega_{2}}\right]$ is contained in a measure zero set coded in $M$; thus we have the consistency of $\neg \mathrm{U}(m)+\neg \mathrm{U}(c)$. A simpler method for producing this result is to start with $M$ a model of $\neg \mathrm{CH}$ and then iteratively with finite support add $\omega_{1}$ random reals. The $\omega_{1}$ random reals $X$ will not have measure zero and the $\omega_{1}$ Cohen reals $Y$ will not be meager; in fact, $X$ will be a Sierpinski set and $Y$ will be a Luzin set.

In [My] Mycielski proves that for any $U \subseteq 2^{\omega} \times 2^{\omega}$ of measure zero there exists a perfect $P$ such that $\forall x, y \in P x \neq y \rightarrow(x, y) \notin U$. We can give a forcing proof of these facts as follows. Let $M$ be a countable transitive model of ZFC (minus power set) containing a code for a Borel set of measure zero covering $U$. Let $Q$ be a perfect set of reals such that $\forall x, y \in Q x \neq y \rightarrow(x, y)$ is $\mathcal{S} \times \mathcal{S}$-generic over $M$, 
and let $r \in 2^{\omega}$ be random over $M$. Then by the prior remarks $\forall x, y \in \mathbf{Q} r$ is random over $M[x, y]$. Let $f: 2^{<\omega} \leftrightarrow \omega$ be $1-1$ in $M$ and define for any $x, r \in 2^{\omega}$, $s(x, r) \in 2^{\omega}$ by $s(x, r)(n)=r(f(x \uparrow n))$. Let $P=\{s(x, r): x \in Q\}$. Then it is easily checked that $P$ is perfect and $\forall x, y \in \mathbf{Q} x \neq y \rightarrow(s(x, r), s(y, r))$ is m.a. $\left(2^{\omega} \times 2^{\omega}\right)$ generic over $M$ (and thus not in $U$ ).

The final goal of this section is to show the consistency of $\mathrm{U}(m)+\mathrm{B}(m)+$ $\neg \mathrm{A}(m)+\neg \mathrm{B}(c)$. In fact we find a partial order $\mathbf{Q}_{\omega_{2}}$ such that $\forall G, \mathbf{Q}_{\omega_{2}}$-generic over $M, M[G] k^{\prime \prime} \mathrm{U}(m)+\mathrm{B}(m)$ " and $\forall f \in M[G] \cap \omega^{\omega} \exists g \in M \cap \omega^{\omega} \forall n f(n)<$ $g(n)$ (recalling Theorems 1.5 and 1.6 we have $\neg \mathrm{A}(m)+\neg \mathrm{B}(c)$ ). Let $\mathrm{B}$ be the measure algebra on $2^{\omega}$ and $\mathbf{P}$ the infinitely often equal order. Let $\mathbf{Q}_{\omega_{2}}$ be the iteration of $\mathbf{B}$ and $\mathbf{P}$ with countable support. That is for any $p \in \mathbf{Q}_{\omega_{2}}$ and for every $\alpha<\omega_{2}$, if $\alpha$ is even or limit then $p\left\lceil\alpha \mid \vdash\right.$ " $p(\alpha) \in \mathbf{B}^{M\left[G_{\alpha}\right] "}$ and if $\alpha$ is odd then

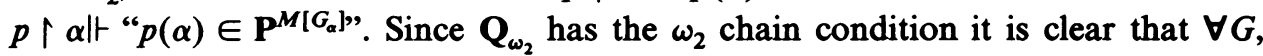
$\mathbf{Q}_{\omega_{2}}$-generic over $M, M[G] k^{\prime \prime} \mathrm{B}(m)+\mathrm{U}(m)$ ". Now we define $p<_{n} q$ for $n<\omega$. If $p, q \in \mathbf{B}$ then $p \leqslant_{n} q$ iff $p<q$ and $\mu(p)>\left(1-1 / 2^{n+1}\right) \mu(q)$. If $p, q \in \mathbf{P}$ then $p<_{n} q \leftrightarrow p<q$ and if $E$ is the first $n$ elements of $\omega-\operatorname{dom}(q)$ then $E \cap \operatorname{dom}(p)$ $=\varnothing$. Now note that if $p_{n+1}<_{n} p_{n} \in \mathrm{B}$ for $n<\omega$ then $\mu\left(\cap\left\{p_{n}: n<\omega\right\}\right)>\frac{1}{2} \mu\left(p_{0}\right)$ and thus $\cap\left\{p_{n}: n<\omega\right\} \in \mathbf{B}$, and also if for each $p_{n} \in \mathbf{P}$ then $\cup\left\{p_{n}: n<\omega\right\} \in$ P. Now define for $F \in\left[\omega_{2}\right]^{<\omega}, n<\omega$, and $p, q \in \mathbf{Q}_{\omega_{2}}\left(p<<_{n}^{F} q\right.$ iff $p<q$ and $\forall \alpha \in F p|\alpha| \vdash$ “ $p(\alpha)<_{n} q(\alpha)$ ”).

LEMMA 7.4. Given $p_{n+1} \leqslant{ }_{n}^{F_{n}} p_{n}$ for $n<\omega$ such that the $F_{n}$ are increasing and $\cup\left\{F_{n}: n<\omega\right\}=\bigcup\left\{\operatorname{supp}\left(p_{n}\right): n<\omega\right\}$ there exists $q \in \mathbf{Q}_{\omega_{2}}$ such that $\forall n q<$ $p_{n}$.

Proof. The proof is entirely similar to Lemma 5 of [L].

LEMMA 7.5. $\forall \alpha \leqslant \omega_{2} \forall \tau \forall p \in \mathbf{Q}_{\alpha} \forall n<\omega \forall F \in\left[\omega_{2}\right]^{<\omega}$,

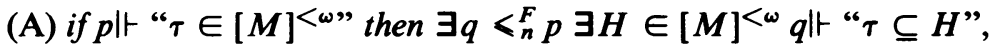

(B) if $p \mid t$ " $\tau \in[M]^{\omega}$ " then $\exists q \leqslant{ }_{n}^{F} p \exists H \in[M]^{\omega} q \Vdash$ " $\tau \subseteq H$ ".

Proof. Note that both of these are easily proved with $\mathbf{P}$ or $\mathbf{B}$ in place of $\mathbf{Q}_{\alpha}$. The proof for $\mathbf{Q}_{\alpha}$ is similar to Lemma 6 of [L] (see also [Ba]).

Using Lemmas 7.4 and 7.5 it is easy to show that if $p$ " $\tau \in \omega^{\omega}$ " then $\exists q<p$ $\exists g \in \omega^{\omega} q \Vdash$ " $\forall n \tau(n)<g(n) "$.

8. The cardinals associated with some of our properties. In this section we investigate the four cardinals $\kappa_{\mathrm{A}}, \kappa_{\mathrm{U}}, \kappa_{\mathrm{B}}$, and $\left|2^{\omega}\right|$. Thus $\kappa_{\mathrm{A}}$ is the least cardinal such that there are meager $X_{\alpha} \subseteq 2^{\omega}$ such that $\cup\left\{X_{\alpha}: \alpha<\kappa_{\mathrm{A}}\right\}$ is not meager, $\kappa_{\mathrm{U}}$ is $\inf \left\{|X|: X \subseteq 2^{\omega}\right.$ not meager $\}$, and $\kappa_{\mathrm{B}}$ is the cardinality of the smallest cover of $2^{\omega}$ by meager sets. Also, for example, the property $A(c)$ (additivity of category) corresponds to $\kappa_{\mathrm{A}}=\left|2^{\omega}\right|$. Note that $\kappa_{\mathrm{A}}$ is an uncountable regular cardinal which is less than or equal to both $\kappa_{U}$ and $\kappa_{B}$ which are both less than or equal to $\left|2^{\omega}\right|$. It is easy to see $\operatorname{cof}\left(\kappa_{U}\right) \geqslant \omega_{1}$ and in [M3] I also show that $\operatorname{cof}\left(\kappa_{B}\right)>\omega_{1}$ (I do not know whether or not the corresponding cardinal for measure can have cofinality $\omega)$.

Considered individually this is all that can be said. Under MA, $\kappa_{A}=\left|2^{\omega}\right|$ (and therefore $\kappa_{\mathrm{A}}$ can be any regular cardinal). If $\kappa$ is any cardinal with $\operatorname{cof}(\kappa)>\omega_{1}$ then 
adding $\kappa$ many Cohen reals (random reals) to a model of $\mathrm{GCH}$ gives a model where $\kappa_{\mathrm{B}}=\kappa\left(\kappa_{\mathrm{U}}=\kappa\right)$. The chart summarizes the known consistency results.

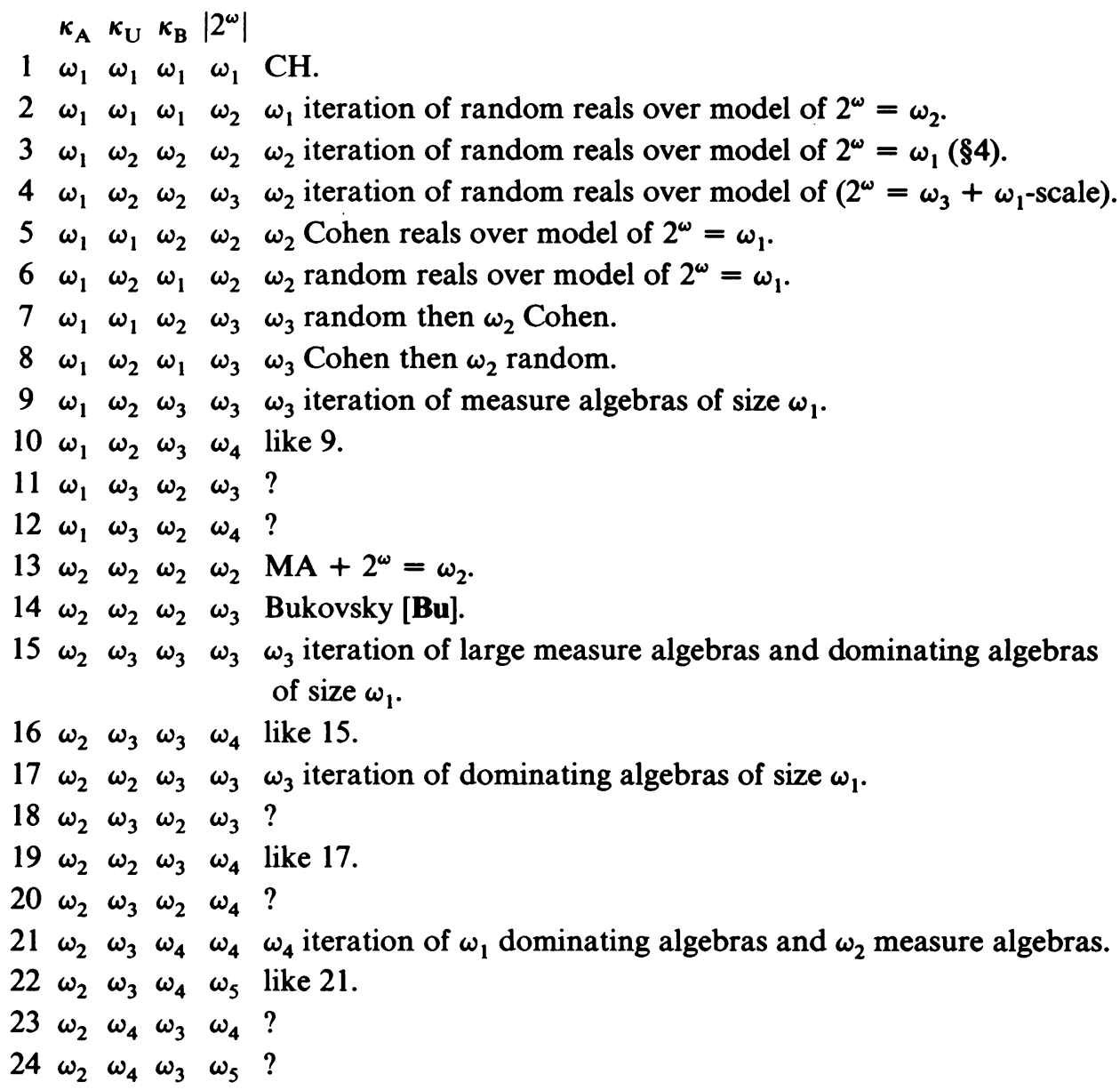

RemarK. Always $\omega_{2}, \omega_{3}, \omega_{4}$, and $\omega_{5}$ can be replaced by any four regular cardinals greater than $\omega_{1}$ in the corresponding order. In 5-8, $\omega_{2}$ and $\omega_{3}$ can be replaced by any cardinals $\kappa_{2}$ and $\kappa_{3}$ such that $\operatorname{cof}\left(\kappa_{2}\right), \operatorname{cof}\left(\kappa_{3}\right)>\omega_{1}$ and $\omega_{2}<\kappa_{2}<\kappa_{3}$. In 2, 4, 14, 19,20 , and $22\left|2^{\omega}\right|$ can be any cardinal of cofinality $>\omega_{1}$. Recall $\mathbf{C}=\{p \mid p: D \rightarrow$ $\left.2, D \in[\omega]^{<\omega}\right\}$ (which adds a Cohen real), B is the measure order-the Borel subsets of $2^{\omega}$ with positive measure (which adds a random real), and $\mathbf{D}=\{(n, f): n<\omega$, $\left.f \in \omega^{\omega}\right\}$ the order for forcing an eventually dominant real (see \$5). All the results in this section will be obtained by iterating various combinations of these orders (except 6 and 7) and in almost all cases we could have used eventually different reals $(\$ 5)$ instead of random reals.

The models. (4) Start with $M F^{\prime \prime} 2^{\omega}=\omega_{3} \wedge \exists D \subseteq \omega^{\omega}|D|=\omega_{1} \forall f \in \omega^{\omega} \exists g \in D$ $\forall n f(n)<g(n)$ ". As in $\S 4$ it is true that if $N$ is an iterated random real extension of $M$ then $\forall f \in N \cap \omega^{\omega} \exists g \in M \cap \omega^{\omega} \exists^{\infty} n f(n)<g(n)$. It follows that $D$ is not eventually dominated in $N$ and so $N k^{*} \kappa_{\mathrm{A}}=\omega_{1}$ " by the results of $\S 1$. It is easy to 
see that $\kappa_{\mathrm{B}} \geqslant \omega_{2}$ and $\kappa_{\mathrm{U}} \geqslant \omega_{2}$ because the iteration has length $\omega_{2}$. Also the set of $\omega_{2}$ Cohen reals added by the iteration is not meager and the set of $\omega_{2}$ random reals does not have measure zero, so $\kappa_{U}=\omega_{2}$ and $\kappa_{\mathrm{B}}=\omega_{2}$ by Rothberger's theorem.

(7) (8) We first prove the following two lemmas.

LemMA 8.1. Suppose $G$ is $\mathbf{C}$-generic over $M$ and $H$ is $\mathbf{B}^{M[G]}$-generic over $M[G]$. Then $M[G, H] \vDash^{*} M[H] \cap 2^{\omega}$ is not meager".

Proof. For any sentence $\theta$ let $\llbracket \theta \rrbracket=\Sigma\{b \in \mathbf{B}: b \Vdash \theta\}$. Suppose $\Vdash$ " $X \in[\omega]^{\omega}$ and $f \in \omega^{\omega}$ " (in the partial order $\mathbf{C} * \tilde{\mathbf{B}}$ ). Note that $\forall p \in \mathbf{C} \forall n<\omega \exists q<p \exists F \in$ $[\omega-n]^{<\omega} \exists\left\langle C_{i}^{m}: i<\kappa_{m}\right\rangle$ a finite sequence of disjoint clopen sets for each $m \in F$ such that

$$
q \Vdash “ \mu\left(\llbracket F \cap X \neq \varnothing \rrbracket \cdot \prod_{m \in F} \llbracket f(m)<k_{m} \rrbracket \cdot \prod_{i<k_{m}}\left(\llbracket f(m)=i \rrbracket \nabla C_{i}^{m}\right)\right) \geqslant 1-\frac{1}{2^{n}} "
$$

where $A \nabla B=(A \cap B) \cup(-A \cap-B)$. So working in $M$ we can build for $k<\omega$, $q_{k} \in \mathbf{C}, n_{k}<n_{k+1}<\omega, F_{k} \in\left[n_{k}, n_{k+1}\right)^{<\omega}$, and $\left(C_{i}^{m}: i<k_{m}\right)$ for $m \in F_{k}$, so that $\forall p \in \mathbf{C} \exists^{\infty} k q_{k} \leqslant p$ and $\forall k q_{k} \Vdash \Vdash \mu\left(B_{k}\right) \geqslant 1-1 / 2^{k+1}$ " where $B_{k}$ is a name for

$$
\llbracket F_{k} \cap X \neq \varnothing \rrbracket \cdot \prod_{m \in F_{k}} \llbracket f(m)<k_{m} \rrbracket \cdot \prod_{i<k_{m}}\left(\llbracket f(m)=i \rrbracket \nabla C_{i}^{m}\right) .
$$

Let $B$ be a name denoting $\cap\left\{B_{k}: q_{k} \in G\right\}$. Then $\vdash_{c}$ “ $\mu(B)>0$ ”. In $M[H]$ define $g(m)=i$ if $H \in C_{i}^{m}$ (0 otherwise), then $(1, B) \Vdash ~ " ~ \exists{ }^{\infty} n \in X f(n)=g(n)$ ". It follows that $\forall f \in M[G, H] \cap \omega^{\omega} \quad \forall X \in M[G, H] \cap[\omega]^{\omega} \quad \exists g \in M[H] \cap \omega^{\omega} \quad \exists^{\infty} n \in X$ $f(n)=g(n)$. By the arguments of $\S 1$ the result follows.

REMARK. Is it true that if we replace $C$ by $D$ in Lemma 8.1 that $M[G, H]$ F"no $x \in 2^{\omega}$ is random over $M[H]$ ]'? If yes this would answer problem (3) of $\S 9$.

LemMA 8.2. Suppose $H$ is B-generic over $M$ and $G$ is $C^{M[H]}=\mathbf{C}$-generic over $M[H]$. Then

$$
\forall f \in M[H, G] \cap \omega^{\omega} \exists g \in M[G] \cap \omega^{\omega} \forall^{\infty} n f(n)<g(n) .
$$

Proof. We may assume $f$ is strictly increasing. By the product lemma, $H$ is $\mathbf{B}^{M}$-generic over $M[G]$. By the proof of Lemma 8.1 (or even a simpler argument) $\forall h \in M[G] \cap M^{\omega} \exists g \in M \exists^{\infty} n h(n)=g(n)$. Working in $M[G]$ obtain $B_{n} \in \mathbf{B}^{M}$ and $g(n)<\omega$ such that $\mu\left(B_{n}\right) \geqslant 1-1 / 2^{n+1}$ and $B_{n} \Vdash$ " $f(n)<g(n)$ ". By above $\exists\left\langle C_{n}: n\langle\omega\rangle \in M\right.$ such that $\exists^{\infty} n C_{n}=B_{n}$ and we may assume $\forall n \mu\left(C_{n}\right)>1$ $1 / 2^{n+1}$. Let $C=\cap_{n<\omega} C_{n}$ and $X=\left\{n: C \subseteq B_{n}\right\}$, then $C \in \mathbf{B}^{M}$ and $X \in M[G]$. Define (in $M[G]) h(n)=g(\inf (X-n))$. Since $f$ is strictly increasing $C \Vdash{ }^{\prime} \forall n$ $f(n)<h(n)$ ".

REMARK. I do not know whether or not $M[G] \cap 2^{\omega}$ has measure zero in $M[H, G]$. This is equivalent to problem (9) of $\S 9$. Note that although it is true that $\forall G$, B-generic over $M, M[G] \vDash^{*} M \cap 2^{\omega}$ does not have measure zero", it is not in general true that if $N \supseteq M$ and $G$ is $\mathbf{B}^{M}$-generic over $N$ then $N[G] F^{\text {" }} N \cap 2^{\omega}$ does not have measure zero". In fact I can show that $N=M\left[G_{0}\right]$, where $G_{0}$ is $\mathbf{B}^{M}$-generic over $M$, is a counterexample. If $\mathbf{C}$ is replaced by $\mathbf{D}$ in Lemma 8.2 does $M[G] \cap \omega^{\omega}$ dominate $M[H, G] \cap \omega^{\omega}$ ? 
Let $\mathbf{B}(\kappa)$ be the measure algebra on $2^{\kappa}$ and $\mathbf{C}(\kappa)=\{p \mid p: D \rightarrow 2$ and $D \in$ $\left.[\kappa]^{<\omega}\right\}$. The model for (7) is $M[G, H]$ where $G$ is $\mathbf{B}\left(\omega_{3}\right)$-generic over $M, H$ is C $\left(\omega_{2}\right)$-generic over $M[G]$, and $M \vDash G C H$. It is easy to see that $\kappa_{\mathrm{B}}>\omega_{2}$ and $\kappa_{\mathrm{U}}=\omega_{1}$ because of $H$. To see that $\kappa_{\mathrm{B}} \leqslant \omega_{2}$ note that by Lemma 8.2 and c.c.c. no real is Cohen over $M[H] \cap 2^{\omega}$. The model for (8) is $M[H, G]$ where $H$ is $\mathbf{C}\left(\omega_{3}\right)$-generic over $M$ and $G$ is $\mathbf{B}^{M[H]}\left(\omega_{2}\right)$-generic over $M[H] . \kappa_{\mathrm{U}}>\omega_{2}$ and $\kappa_{\mathrm{B}}=\omega_{1}$ because of $G$. By Lemma 8.1 and c.c.c. $M[G] \cap 2^{\omega}$ is not meager so $\kappa_{\mathrm{U}}<\omega_{2}$.

The remaining models will all be obtained by c.c.c. finite support iterations (of length $\kappa_{\mathrm{B}}$ ). Suppose $M \vDash Z F C$ and $\mathbf{P}_{\alpha}$ for $\alpha \leqslant \gamma$ is such an iteration. Recall $\mathbf{P}_{\alpha+1}=\mathbf{P}_{\alpha} * \mathbf{P}^{\alpha}$ where $\mathbf{P}^{\alpha}$ is a term in the forcing language of $\mathbf{P}_{\alpha}$. Suppose $\Sigma \subseteq \gamma$ and $\Sigma \in M$; then define $\mathbf{P}_{\alpha}^{*}=\left\{p \in \mathbf{P}_{\alpha}: \operatorname{supp}(p) \subseteq \Sigma\right\}$.

Lemma 8.3. Suppose $\forall \alpha<\gamma \mathbf{P}^{\alpha}$ is a term in the forcing language of $\mathbf{P}_{\alpha}^{*}$. Then $\forall \alpha \leqslant \gamma$ if $G_{\alpha}$ is $\mathbf{P}_{\alpha}$-generic over $M$ then $G_{\alpha} \cap \mathbf{P}_{\alpha}^{*}$ is $\mathbf{P}_{\alpha}^{*}$-generic over $\boldsymbol{M}$.

Proof. The proof is by induction on $\alpha$. For $\alpha$ a limit ordinal this is proved exactly as Lemma 5.7. Also for the case $\alpha+1$ if $\alpha \notin \Sigma$ then $\mathbf{P}_{\alpha+1}^{*}$ is isomorphic to $\mathbf{P}_{\alpha}^{*}$. If $\alpha \in \Sigma$ then $\mathbf{P}_{\alpha} \in M\left[G_{\alpha} \cap \mathbf{P}_{\alpha}^{*}\right]$ and $G_{\alpha+1} \cap \mathbf{P}_{\alpha+1}^{*}=\left(G_{\alpha} \cap \mathbf{P}_{\alpha}^{*}\right) \times G^{\alpha}$; so by the product lemma $G_{\alpha+1} \cap \mathbf{P}_{\alpha+1}^{*}$ is $\mathbf{P}_{\alpha+1}^{*}$-generic over $M$.

LemMa 8.4. Suppose $\kappa$ is an uncountble cardinal and $G \times H$ is $\mathbf{C}(\kappa) * P$-generic over $M$ where $\mathbf{P} \in M[G \uparrow \Sigma]$ for some $\Sigma \subseteq \kappa, \Sigma \in M$, and $|\Sigma|<\kappa$. Then $M[G] \cap$ $2^{\omega}$ is not meager in $M[G, H]$.

Proof. This is immediate from the product lemma, since $G \uparrow(\kappa-\Sigma)$ is $\mathrm{C}(\kappa-\Sigma)$-generic over $M[G \uparrow \Sigma, H]$.

(9) Start with $M \vDash G C H$ and let $\Sigma_{\alpha} \subseteq \alpha$ for $\omega_{2}<\alpha<\omega_{3}$. List all subsets of $\omega_{3}$ of cardinality $\omega_{1}$. Let $\mathbf{P}_{\alpha}$ for $\alpha \leqslant \omega_{3}$ be the following iteration.

For $\alpha<\omega_{2}$ let $\mathbf{P}_{\alpha+1}=\mathbf{P}_{\alpha} * \mathbf{C}$.

For $\omega_{2}<\alpha<\omega_{3}$ let $\mathbf{P}_{\alpha+1}=\mathbf{P}_{\alpha} * \mathbf{B}^{M\left[G_{\alpha}^{*}\right]}$ where $G_{\alpha}^{*}=G_{\alpha} \cap\{p \in$ $\left.\mathbf{P}_{\alpha}: \operatorname{supp}(p) \subseteq \Sigma_{\alpha}\right\}$. Since the length of this iteration is $\omega_{3}$ it is easy to see that $\kappa_{B}=\omega_{3}=\left|2^{\omega}\right|$. Since "partially" random reals are added, $\kappa_{U}>\omega_{2}$. Also the arguments of $\S 4$ easily show that $M \cap \omega^{\omega}$ is not eventually dominated so $\kappa_{\mathrm{A}}=\omega_{1}$.

By Lemmas 8.3 and $8.4, M\left[G_{\omega_{2}}\right] \cap 2^{\omega}$ is not meager in $M\left[G_{\omega_{3}}\right]$. This is because for any $\tau$ a term such that $\mathbb{F}_{\omega_{3}}$ “ $\tau \subseteq \omega$ " (or even $\mathbb{t}$ " $\tau \subseteq \omega_{1}$ "), by using c.c.c. we can find $\Sigma \subseteq \omega_{3},|\Sigma|=\omega_{1}, \Sigma \in M, \forall \alpha \in \Sigma, \Sigma_{\alpha} \subseteq \Sigma$, and $\tau$ is a term in the forcing language of $\mathbf{P}_{\omega_{3}}^{*}=\left\{p \in \mathbf{P}_{\omega_{3}}: \operatorname{supp}(p) \subseteq \Sigma\right\}$. Thus $\mathbf{P}_{\omega_{3}}^{*} \in M\left[G \uparrow \Sigma \cap \omega_{2}\right]$ so Lemma 8.4 applies.

(10) Let $M k^{\prime \prime} 2^{\omega}=\omega_{4}$ " be such that $M k^{*} \exists D \subseteq \omega^{\omega}|D|=\omega_{1} \forall f \in \omega^{\omega} \exists g \in D$ $\forall n f(n)<g(n)$ and $\exists \Sigma_{\alpha} \subseteq \alpha$ for $\alpha<\omega_{3}$ with $\left|\Sigma_{\alpha}\right|=\omega_{1}$ and $\forall A \subseteq \omega_{3}$ if $|A|=\omega_{1}$ then $\exists \alpha \Sigma_{\alpha} \subseteq A$ ".

For example this holds if $M$ is a random real $\left(\mathbf{B}\left(\omega_{4}\right)\right)$ extension of a model of GCH. Now do the same iteration as in (9). To see that $\kappa_{B}<\omega_{3}$ note that

$$
2^{\omega}=\bigcup\left\{2^{\omega} \cap M\left[G_{\alpha}^{*}\right]: \alpha<\omega_{3}\right\}
$$

and $2^{\omega} \cap M\left[G_{\alpha}^{*}\right]$ is meager for each $\alpha<\omega_{3}$. Also $\kappa_{\mathrm{A}}=\omega_{1}$ since $D$ is not eventually dominated. 
(14) This is due to Bukovsky [Bu] who does it by starting with a model of $2^{\omega}=\omega_{3}$ and then doing an $\omega_{2}$ iteration. At each step of the iteration he does an $\omega_{3}$ iteration making MA true. Alternatively we could start with a model of $2^{\omega}=\omega_{3}$ and then do an $\omega_{2}$ iteration with $\mathbf{D}$.

(15) Let $M$ F" GCH and $\Sigma_{\alpha} \subseteq \alpha$ for $\omega_{2}<\alpha<\omega_{3}$ and $\alpha$ even, list all subsets of $\omega_{3}$ of size $\omega_{1}$ ". For $\alpha<\omega_{2}$ let $\mathbf{P}^{\alpha}=\mathbf{C}$. For $\omega_{2}<\alpha<\omega_{3}$ and $\alpha$ even, let $\mathbf{P}^{\alpha}=\mathbf{D}^{M\left[G_{\alpha}^{*}\right]}$ where $G_{\alpha}^{*}=\left\{p \in G_{\alpha}: \operatorname{supp}(p) \subseteq \Sigma_{\alpha}\right\}$. For $\alpha$ odd, let $\mathbf{P}^{\alpha}=\mathbf{B}^{M\left[G_{\alpha}\right]}$. It is easy to check that $\kappa_{\mathrm{U}}=\kappa_{\mathrm{B}}=\omega_{3}=\left|2^{\omega}\right|$ and $\kappa_{\mathrm{A}} \geqslant \omega_{2}$. We claim that $M\left[G_{\omega_{2}}\right] \cap \omega^{\omega}$ is not eventually dominated (and thus by $\S 1 \kappa_{A}=\omega_{2}$ ).

Lemma 8.5. Suppose $G$ is $\mathbf{C}\left(\omega_{1}\right)$-generic over $M, \exists \gamma \exists X \subseteq \gamma X \in M$, and $\mathbf{P}_{\alpha}$ for $\alpha \leqslant \gamma$ a finite support iteration over $M[G]$ such that $\forall \alpha \in X \mathbf{P}^{\alpha}$ is a (canonical) name for a partial order in $M$ and $\forall \alpha \in \gamma-X \mathbf{P}^{\alpha}$ is a name for a boolean algebra which has a finitely additive positive measure on it (for short call it a measure algebra). Then $M[G] \cap \omega^{\omega}$ is not dominated in $M[G, H]$ when $H$ is $\mathbf{P}_{\gamma}$-generic over $M[G]$.

Proof. Note that $\mathbf{P}_{\gamma} \simeq\left(\Sigma_{\alpha \in X} \mathbf{P}^{\alpha}\right) * \mathbf{P}^{1}$ where $\Sigma$ is the direct sum and $\mathbf{P}^{1}$ is a name for some iteration of measure algebras. Thus $H=H_{1} \times H_{2}$ and by the product lemma $G$ is $\mathbf{C}\left(\omega_{1}\right)$-generic over $M\left[H_{1}\right]$. Note that $\mathbf{C}\left(\omega_{1}\right) * \mathbf{P}^{1}$ is also an iteration of measure algebras-i.e. regard $C\left(\omega_{1}\right)$ as the $\omega_{1}$ iteration of the two element boolean algebra $\{0,1\}$. Now suppose $f \in \omega^{\omega} \cap M\left[H_{1}, G, H_{2}\right]$. Working in $M\left[H_{1}\right]$ and using the arguments of $\S 4$ we can find $p_{n}$ in $\mathbf{C}\left(\omega_{1}\right) * \mathbf{P}^{1}$ for $n<\omega$ and $h \in \omega^{\omega} \cap M\left[H_{1}\right]$ so that $p_{n} \Vdash$ " $f(n)<h(n)$ " and for any $p$ in $\mathbf{C}\left(\omega_{1}\right) * \mathbf{P}^{1} \exists n_{0}$ $\forall n>n_{0} p_{n}$ and $p$ are compatible and in fact $\exists q<p_{n} q<p, \operatorname{supp}(q) \subseteq \operatorname{supp}(p) \cup$ $\operatorname{supp}\left(p_{n}\right)$. Choose $\alpha<\omega_{1}$ so that $\forall n<\omega(\alpha+n) \notin U\left\{\operatorname{supp}\left(p_{n}\right): n<\omega\right\}$ and define $g \in \omega^{\omega}$ by $g(n)=$ least $m \geqslant n$ such that $G(\alpha+m)=1$. Then $g \in M[G] \cap$ $\omega^{\omega}$ and $\exists^{\infty} n g(n) \geqslant f(n)$. To see this suppose not and $p \Vdash$ " $\forall n>n_{0} g(n)<f(n)$ "; then $\exists n_{1}>n_{0}$ such that $\forall n \geqslant n_{1} \alpha+n \notin \operatorname{supp}(p)$ and $\exists q<p q<p_{n}$ and $\forall n>$ $n_{1}(\alpha+n) \notin \operatorname{supp}(q)$. Extend $q$ to $q^{\prime}$ by letting $q^{\prime}(\beta)=0$ for all $\beta$ such that $\alpha+n_{1}<\beta \leqslant \alpha+h\left(n_{1}\right)$; then $q^{\prime} \mid$ " $~ f\left(n_{1}\right)<g\left(n_{1}\right)$ ".

The problem may be reduced to the lemma by an argument combining the ideas of Lemmas 8.3 and 5.7. First reduce to $\exists \gamma<\omega_{3} \exists \mathbf{P}_{\gamma}$ finite support iteration over $M$ such that $\left|\gamma-\omega_{2}\right|<\omega_{1}$ and $\forall \alpha<\omega_{2} \mathbf{P}^{\alpha}=\mathbf{C}$ and $\forall \alpha \omega_{2}<\alpha<\gamma$ and either $\mathbf{P}^{\alpha}$ is $\mathbf{D}^{M\left[G_{\alpha}^{*}\right]}$ where $G_{\alpha}^{*}=\left\{p \in G_{\alpha}: \operatorname{supp}(p) \subseteq \Sigma_{\alpha}\right\}, \Sigma_{\alpha} \subseteq \alpha,\left|\Sigma_{\alpha}\right|<\omega_{1}, \Sigma_{\alpha} \in M$ or $\mathbf{P}^{\alpha}$ is $\mathbf{B}^{M\left[G_{\alpha}\right]}$. By swallowing up $G \uparrow \Sigma_{\alpha} \cap \omega_{2}$ for $\omega_{2}<\alpha<\gamma$ we reduce to the lemma.

(16) Let $M k^{\prime \prime} 2^{\omega}=\omega_{4}$ " in which there exists $\Sigma_{\alpha} \subseteq \alpha$ for $\alpha<\omega_{3}$ with $\left|\Sigma_{\alpha}\right|<\omega_{1}$ and $\forall A \subseteq \omega_{3}|A|=\omega_{1} \rightarrow \exists \alpha A \subseteq \Sigma_{\alpha}$. Proceed as in (15). Note that because of forcing with $B^{M\left[G_{\alpha}\right]}$ for $\alpha$ odd $2^{\omega}$ is the union of $\omega_{3}$ meager sets so $\kappa_{B}<\omega_{3}$.

(17) Let $M \vDash$ "GCH" and let $\Sigma_{\alpha} \subseteq \alpha$ for $\omega_{2}<\alpha<\omega_{3}$; list all subsets of $\omega_{3}$ of size $\omega_{1}$. Let $\mathbf{P}_{\alpha+1}=\mathbf{P}_{\alpha} * \mathbf{C}$ for $\alpha<\omega_{2}$ and let $\mathbf{P}_{\alpha+1}=\mathbf{P}_{\alpha} * \mathbf{D}^{M\left[G_{\alpha}^{*}\right]}$ where as before $G_{\alpha}^{*}=G_{\alpha} \cap\left\{p \in \mathbf{P}_{\alpha}: \operatorname{supp}(p) \subseteq \Sigma_{\alpha}\right\}$.It is easily seen that $\kappa_{\mathrm{B}}=\omega_{3}=\left|2^{\omega}\right|$ and $\kappa_{\mathrm{A}} \geqslant \omega_{2}$. By Lemmas 8.3 and 8.4 it is not hard to see that $M\left[G_{\omega_{2}}\right] \cap 2^{\omega}$ is not meager, so $\kappa_{\mathrm{A}}=\kappa_{\mathrm{U}}=\omega_{2}$.

(19) This is just like (17) only start with $M k^{"} 2^{\omega}=\omega_{4}$ and $\exists \Sigma_{\alpha} \subseteq \alpha$ for $\alpha<\omega_{3}$, $\left|\Sigma_{\alpha}\right|=\omega_{1}$ and $\forall A \subseteq \omega_{3}|A| \leqslant \omega_{1} \rightarrow \exists \alpha A \subseteq \Sigma_{\alpha} "$ 
(21), (22) Working in $M$ let $\Sigma_{\alpha} \subseteq \alpha$ for $\alpha<\omega_{4}$ be a list such that for all $\alpha$ even, $\left|\Sigma_{\alpha}\right|<\omega_{1}$ and for all $\alpha$ odd, $\left|\Sigma_{\alpha}\right| \leqslant \omega_{2}$ such that for every $A \subseteq \omega_{4},\left(|A|<\omega_{1} \rightarrow \exists \alpha\right.$ even $\left.A \subseteq \Sigma_{\alpha}\right)$ and $\left(|A| \leqslant \omega_{2} \rightarrow \exists \alpha\right.$ odd $\left.A \subseteq \Sigma_{\alpha}\right)$. Let $\mathbf{P}_{\omega_{4}}$ be the $\omega_{4}$ iteration defined by letting

$$
\mathbf{P}^{\alpha}=\mathbf{D}^{M\left[G_{\alpha}^{*}\right]} \text { for } \alpha \text { even }
$$

and

$$
\mathbf{P}^{\alpha}=\mathbf{B}^{M\left[G_{\alpha}^{*}\right]} \quad \text { for } \alpha \text { odd }
$$

where

$$
G_{\alpha}^{*}=\left\{p \in G_{\alpha}: \operatorname{supp}(p) \subseteq \Sigma_{\alpha}\right\} .
$$

Easily we have in $M\left[G_{\omega_{4}}\right]$ that $\kappa_{\mathrm{A}}>\omega_{2}, \kappa_{\mathrm{U}}>\omega_{3}$ and $\kappa_{\mathrm{B}}>\omega_{4}$. In case $M \vDash \mathrm{FCH}$ we have immediately that $\kappa_{B}=\omega_{4}$; in any case

$$
M\left[G_{\omega_{4}}\right] \cap 2^{\omega}=\cup\left\{M\left[G_{\alpha}^{*}\right] \cap 2^{\omega}: \alpha<\omega_{4}\right\}
$$

and each $M\left[G_{\alpha}^{*}\right] \cap 2^{\omega}$ is meager. To see that $\kappa_{U}<\omega_{3}$ use Lemma 8.3 and to see that $\kappa_{\mathrm{A}}<\omega_{2}$ use an argument similar to the one used in (15).

\section{Problems. ${ }^{2}$}

(1) Show that $\mathrm{A}(m)$ does not imply $\mathrm{A}(c)$.

(2) Show that $\neg \mathrm{A}(m)+\mathrm{B}(m)+\mathrm{A}(c)$ is consistent. The natural model for this might be obtained by iteratively adding (with finite support) $\omega_{2}$ dominating reals and random reals.

(3) Show that $\mathrm{U}(c)+\neg \mathrm{U}(m)+\neg \mathrm{B}(m)$ is consistent. I conjecture that this holds if one adds $\omega_{2}$ eventually dominating reals and then $\omega_{1}$ random reals.

(4) (Fremlin) Show that the least $\kappa\left(\kappa_{\mathrm{B}}^{m}\right)$ such that $2^{\omega}$ can be covered by $\kappa$-many measure zero sets cannot have countable cofinality (see [MB] for the ideal of meager sets).

(5) (Baumgartner) Show that if one adds a Laver real (see [L]) the ground reals have measure zero.

(6) Show that $X$ is necessary in Theorem 1.3.

(7) Show that $\forall i<n f(i)<n$ is necessary in Theorem 1.4.

(8) Find characterizations of $U(m), B(m)$, and $A(m)$.

(9) Suppose $B \subseteq 2^{\omega} \times 2^{\omega} \times 2^{\omega}$ is a Borel set such that

$$
\forall x \forall y \mu(\{z:(x, y, z) \in B\})=0 .
$$

Then does there exist a Borel function $F: 2^{\omega} \rightarrow 2^{\omega}$ such that $\exists A \subseteq 2^{\omega} \mu(A)>0$ $\forall x \in A\{y:(x, y, F(y)) \notin B\}$ is not meager?

\section{REFERENCES}

[Ba] J. Baumgartner, Set theory notes, Cambridge Summer School, 1978.

[BL] J. Baumgartner and R. Laver, Iterated perfect-set forcing, Ann. Math. Logic 17 (1979), 271-288.

[Bu] L. Bukovský, Random forcing, Set Theory and Hierarchy Theory. V, Lecture Notes in Math., Vol. 619, Springer-Verlag, Berlin and New York, 1977.

[FS] D. Fremlin and S. Shelah, On partitions of the real line, Notices Amer. Math. Soc. 25 (1978), A-600.

[G] S. Grigorieff, Combinatorics on ideals and forcing, Ann. Math. Logic 3 (1971), 363-394.

${ }^{2}$ The bias expressed here is the author's. 
[H] S. H. Heckler, On the existence of certain cofinal subsets of $\omega^{\omega}$, Axiomatic Set Theory, Proc. Sympos. Pure Math., vol. 13, Amer. Math. Soc., Providence, R. I., 1974, pp. 155-174.

[I] J. Shinoda, A note on Silver's extension, Comment. Math. Univ. St. Paul. 22 (1970), 109-111.

[K1] K. Kunen, "Toronto talks", 1975.

[K2] , Set theory, North-Holland, Amsterdam, 1981.

[Ku] K. Kurawtowksi, Topology, Vol. 1, Academic Press, New York, 1966.

[L] R. Laver, On the consistency of Borel's conjecture, Acta Math. 137 (1976), 151-169.

[Ma] A. R. D. Mathias, Happy families, Ann. Math. Logic 12 (1977), 59-111.

[MS] D. A. Martin and R. M. Solovay, Internal Cohen extensions, Ann. Math. Logic 2 (1970), 143-178.

[M1] A. W. Miller, Covering $2^{\omega}$ with $\omega_{1}$ disjoint closed sets, The Kleene Symposium, North-Holland, Amsterdam, 1980, pp. 415-421.

[M2] _ On the length of Borel hierarchies, Ann. Math. Logic 16 (1979), 233-267.

[M3] _ The Baire category theorem and cardinals of countable cofinaity, J. Symbolic Logic (to appear).

[My] J. Mycielski, Algebraic independence and measure, Fund. Math. 61 (1967), 165-169.

[O] J. C. Oxtoby, Measure and category, Springer-Verlag, Berlin and New York, 1970.

[R1] F. Rothberger, Eine Aquivalenz zwischen der Kontinumbypothese under der Existenz der Lusinschen und Sierpinskischen Mengen, Fund. Math. 30 (1938), 215-217.

[R2] _ Sur les familles indenombrables de suites de nombres naturels et les problèmes concernant la propriété C, Proc. Cambridge Philos. Soc. 37 (1941), 109-126.

[R3] _ Sur un ensemble toujours de première catégorie qui est déprourou de la propriété $\lambda$, Fund. Math. 32 (1939), 294-300.

[R4] __, On some problems of Hausdorff and of Sierpinski, Fund. Math. 35 (1948), 29-46.

[R5] __, On families of real functions with a denumerable base, Ann. of Math. 45 (1944), 397-406.

[R6] __ Eine Verschärfung der Eigenschaft C, Fund. Math. 30 (1938), 50-55.

[Ru] M. E. Rudin, Martin's Axiom, Handbook of Mathematical Logic, North-Holland, Amsterdam, 1977, pp. 491-502.

[Sa] G. E. Sacks, Forcing with perfect closed sets, Axiomatic Set Theory, Proc. Sympos. Pure Math., vol. 13, Amer. Math. Soc., Providence, R. I., 1970, pp 331-356.

[Sh] J. R. Shoenfield, Unramified forcing, Axiomatic Set Theory, Proc. Sympos. Pure Math., vol. 13, Amer. Math. Soc., Providence, R. I., 1970, pp. 357-382.

[So] R. M. Solovay, A model of set theory in which every set of reals is Lebesgue measurable, Ann. of Math. (2) 92 (1970), 1-56.

[ST] R. M. Solovay and S. Tennenbaum, Iterated Cohen extensions and Souslin's problem, Ann. of Math. 94 (1971), 201-245.

[T] J. Truss, Sets having calibre $\aleph_{1}$, Studies in Logic and the Foundations of Math., vol. 87, North-Holland, Amsterdam, 1977.

Department of Mathematics, University of Wisconsin, Madison, Wisconsin 53706 